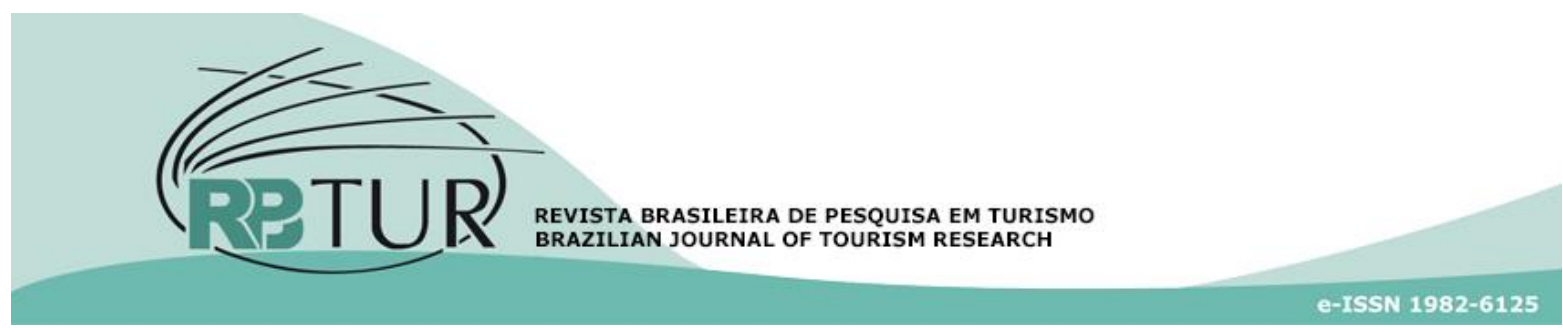

\title{
Artículo
}

\section{Modelamiento y proyección de la demanda de turismo internacional en Puno-Perú}

\author{
Modelling and forecasting international tourism demand in Puno-Peru \\ Modelagem e previsão da procura por turismo internacional em Puno- \\ Peru
}

\section{Luis Francisco Laurente Blanco; Ronald Wilson Machaca Hancco ${ }^{1}$}

1 Universidad Nacional de Altiplano, Puno, Peru

Palavras clave:

Estacionalidad; lago Titicaca;

Perú;

ARIMA;

Cultura.

\section{Keywords:}

Seasonality;

Titicaca lake;

Peru;

ARIMA;

Culture.

\section{Resumen}

La industria del turismo en el Perú genera cerca de 1.1 millones de puestos de trabajo y aporta el 3.3\% del PBI, lo que la convierte en una de sus principales actividades económicas, de esta forma el turismo deja de ser sólo una actividad comercial y se transforma en una herramienta para el desarrollo de la población peruana especialmente en las regiones con alta tasa de pobreza y con numerosos atractivos turísticos como es el caso de la región de Puno con una tasa de pobreza de $24.2 \%$ que está ubicada en el sur del país y que cuenta con numerosos atractivos turísticos de tipo naturales, históricos, culturales y gastronómicos. El objetivo de esta investigación es modelar y proyectar la demanda de turistas internacionales que visitan Puno utilizando la metodología ARIMA de Box-Jenkins, para ello el estudio considera información mensual de arribo de turistas internacionales entre los años 2003 a 2017. Finalmente, utilizando los estadísticos MAPE, Z, r, Criterio de Información de Akaike (AIC) y Criterio de Schwarz (SC) se identificó al modelo SARIMA $(6,1,24)(1,0,1) 12$ como el más eficiente para el modelamiento y proyección de la demanda del turismo internacional en la región de Puno.

\section{Abstract}

The tourism industry in Peru generates about 1.1 million jobs and contributes 3.3\% of GDP, which makes it one of its main economic activities, so tourism is no longer just a commercial activity and transforms into a tool for the development of the Peruvian population especially in regions with high poverty rate and with numerous tourist attractions as it is the case of the Puno region with a poverty rate of $24.2 \%$ that is located in the south of the country and that has numerous tourist attractions of natural, historical, cultural and gastronomic type. The objective of this research is to model and forecasting the demand of international tourists visiting Puno using the ARIMA methodology of Box-Jenkins, for this the study considers monthly arrival information of foreign tourists between the years 2003 to 2017 . Finally, using the statistics MAPE, Z, r, Akaike Information Criterion (AIC) and Schwarz Criterion (SC) was identified to the SARIMA $(6,1,24)(1,0,1) 12$ model as the most efficient for modeling and forecasting of the demand for international tourism in the Puno region. 
Palavras-chave:

Sazonalidade;

lago Titicaca;

Peru;

ARIMA;

Cultura.

Revisado por pares.

Recibido en: 05/02/2019.

Aprobado en: 09/04/2019.

\begin{abstract}
Resumo
A indústria do turismo no Peru gera aproximadamente 1.1 milhão de empregos e contribui com 3.3\% do PIB, o que a torna uma de suas principais atividades econômicas, portanto o turismo não é mais apenas uma atividade comercial mas é uma ferramenta para o desenvolvimento da população peruana, especialmente nas regiões com alto índice de pobreza e muitas atrações turísticas como é o caso da região de Puno com uma taxa de pobreza de $24.2 \%$ localizada no sul do país e com muitas atrações históricas, naturais, cultural e gastronômico. 0 objetivo desta pesquisa é modelar a procura de turistas internacionais que visitam Puno utilizando a metodologia ARIMA de Box-Jenkins, para este estudo considera informações mensais de chegadas de turistas internacionais entre os anos 2003 e 2017. Finalmente, usando estatísticas MAPE, Z, R, Critério de Informação de Akaike (AIC) e Critério de Schwarz (SC) se encontrou ao modelo SARIMA $(6,1,24)(1,0,1) 12$ como o mais eficiente para a modelação e previsão da procura do Turismo Internacional na região de Puno.
\end{abstract}

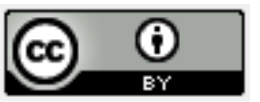

Como citar: Blanco, L. F. L.; Hancco, R. W. M. (2020). Modelamiento y proyección de la demanda de turismo internacional en Puno-Perú. Revista Brasileira de Pesquisa em Turismo, São Paulo, 14 (1), p. 34-55, jan./abr. http://dx.doi.org/10.7784/rbtur.v14i1.1606

\section{INTRODUCCIÓN}

Considerando los recursos naturales, cultura, gastronomía, folclore, historia, entre otros, la industria del turismo es cada vez más importante en la economía de los países ya que está estrechamente relacionado al desarrollo social y económico. Según la Organización Mundial del Turismo (OMT), el turismo ha crecido con mayor rapidez en los últimos años ubicándose como tercera categoría de exportación estando por detrás de los productos químicos y combustibles y por delante de productos de automoción y alimentación, de este modo, las llegadas de turistas internacionales en el mundo pasaron de 674 millones en el año 2000 a 1,235 millones en 2016 y los ingresos registrados por los destinos de todo el mundo pasaron de 495,000 millones de dólares en el año 2000 a 1.22 billones de dólares en 2016 (OMT, 2017).

En el Perú esta industria genera cerca de 1.1 millones de puestos de trabajo y aporta el 3.3\% del PBI (Camara, 2018) donde en el año 2017 el PBI ascendió a un valor de 157,744 millones de dólares donde el sector turismo representa el $3.2 \%$ de este total encontrándose por encima de los sectores pesca, acuicultura, electricidad y gas natural y presentando un crecimiento de 1.4\% respecto de 2016 (Banco Central de Reserva del Perú [BCRP], 2018), lo que convierte al sector turismo en una de sus principales actividades económicas debido que durante el mismo año arribaron al país 4 millones 32 mil 339 turistas internacionales que representa un crecimiento del $8 \%$ en el turismo receptivo respecto a lo alcanzado en 2016 (MINCETUR, 2017a) donde los principales países que visitaron el Perú en el año 2017 fueron: Chile (27\%), Estados Unidos $(15 \%)$, Venezuela (5\%), Ecuador (7\%), Colombia (5\%) y Argentina $(5 \%)$ haciendo una participación del mercado de $69 \%$ de llegadas al país (GESTION, 2017). Los principales puntos de ingreso al país fueron: Aeropuerto Internacional Jorge Chávez (58\%), Tacna (23\%), Tumbes (9\%) y Puno (5\%) (MINCETUR, 2017b). Se estima que los ingresos de divisas generados por el turismo receptivo en el Perú, durante el año 2017, alcanzaron los 4,574 millones de dólares, representando un crecimiento del 6\% en relación al año 2016 (MINCETUR, 2017b).

En los últimos años, en el país se ha apostado por un turismo sostenible que promueve políticas, prácticas y comportamientos éticos a través de esta actividad mediante el uso eficiente de los recursos; asimismo, se ha buscado fomentar la paz, el desarrollo y la erradicación de la pobreza. De esta forma, el turismo deja de ser sólo una actividad comercial y se transforma en una herramienta para el desarrollo de la población peruana especialmente en las regiones con mayor tasa de pobreza y con numerosos atractivos turísticos como es el caso de la región de Puno, cuarta región más visitada por los turistas internacionales (véase Figura 1), que a la fecha tiene una tasa de pobreza de $24.2 \%$, ubicándola en la décima región más pobre del Perú (INEl, 2018a) y sin embargo está dotada de numerosos atractivos turísticos que podría en el futuro ser explotados con mayor eficiencia con políticas de turismo sostenible.

En el año 2017 el PBI de la región de Puno fue más de 2,892 millones de dólares que representa una variación de $3.9 \%$ respecto del año 2016 , donde el sector turismo representa el $2 \%$ del PBI regional que 
registró visitas internacionales por más de 62.5 millones de dólares en el mismo año y que se encuentra por arriba del sector pesca, electricidad y gas con un crecimiento en 2017 de 2.43\% respecto de 2016. Asimismo, el crecimiento anual del sector turismo en Puno desde 2010 es siempre positivo al igual que el sector agropecuario que es el sector más representativo del PBI de Puno (INEI, 2018b).

En la actualidad, la actividad turística en Puno es de importancia porque de ella se benefician cientos de personas, es así que el sector turismo en la región de Puno en el 2017 generó más de 90 mil puestos de trabajo; y se estima mediante la Cámara Nacional de Turismo (CANATUR) que el turismo en el 2035 será uno de los primeros sectores que generará el desarrollo e incrementará el empleo en la región de Puno (CORREO, 2017); de ahí la importancia para las empresas del sector tener una buena proyección sobre el número de arribo de turistas internacionales, para de ese modo realizar una mejor planificación, previsión y administración de la actividad.

Figura 1 - Arribo de visitantes internacionales a establecimientos de hospedaje, según regiones del Perú, 2003-2016

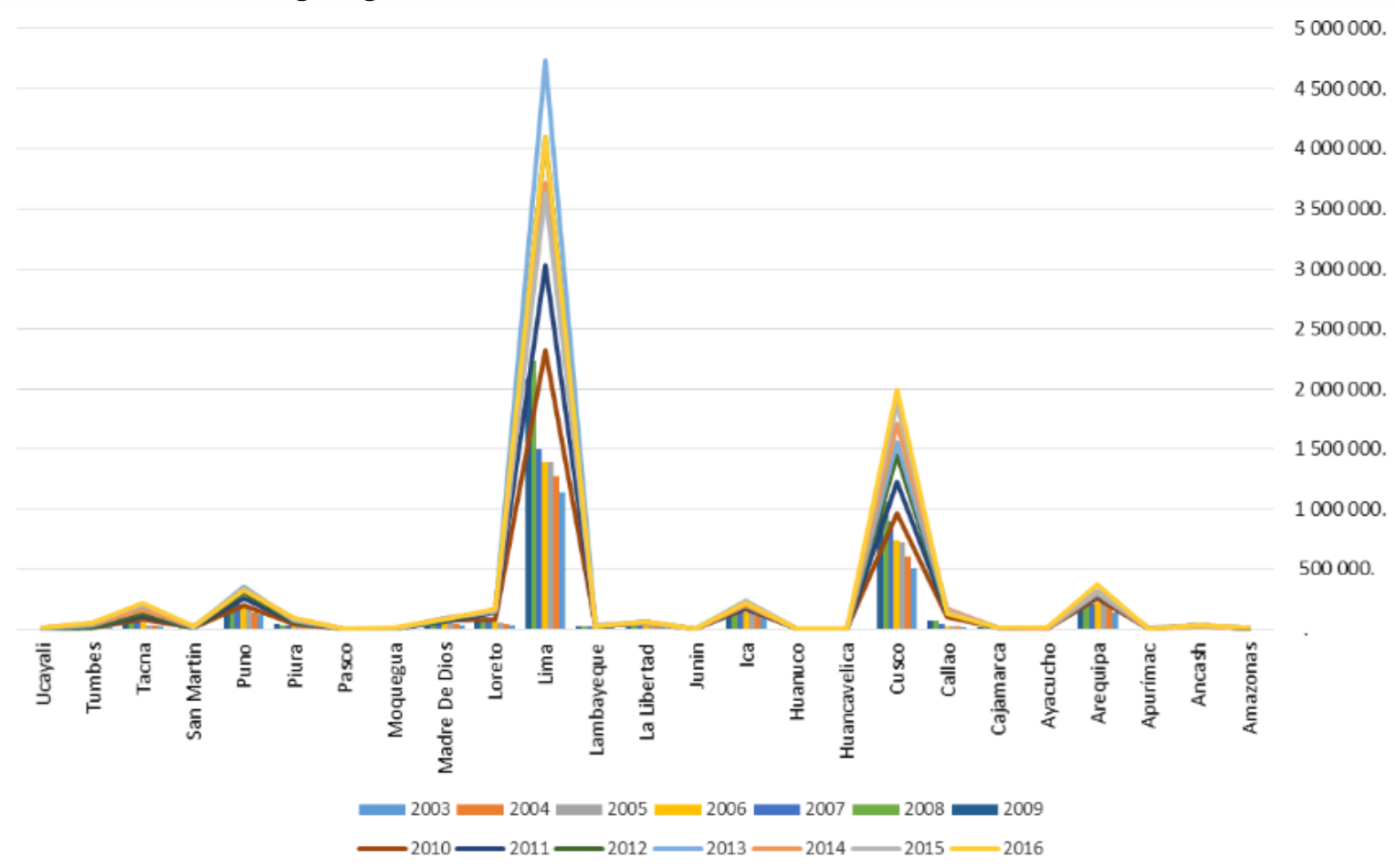

Fuente: Elaboración propia en base a información de MINCETUR

Como una breve descripción, la región de Puno se encuentra ubicada en la zona sur del Perú a orillas del lago Titicaca (denominado el "lago navegable más alto del mundo" (INRENA, 1995) a una altitud de 3,827 msnm con un clima frío y seco y es considerada como un buen destino turístico debido a la infraestructura, servicios básicos, ubicación, presencia de diversos escenarios naturales (Cayo \& Apaza, 2017) y por la creación de nuevas modalidades de hacer turismo en la región como es el caso del turismo ecológico, turismo rural, turismo de aventura, turismo vivencial y otras modalidades del llamado turismo alternativo principalmente en las comunidades de Amantaní, Pucará, Llachón, Anapia, Atuncolla y Sillustani donde los visitantes pueden convivir por unos días en estas comunidades aprendiendo más sobre sus tradiciones y costumbres (Mamani, 2016). Como principales atractivos turísticos cuenta con: el lago Titicaca, malecón eco turístico Bahía de los Incas, isla flotante de los Uros (Figura 2), isla Amantaní, isla Taquile, Llachón, entre otros (Puno, 2017). Por otro lado, la región de Puno ofrece una diversidad de destinos turísticos de tipo histórico-culturales entre ellos restos arqueológicos en diversas ciudades y cuenta con una vasta diversidad en los recursos folklórico-culturales. Asimismo, la región cuenta con una amplia variedad de recursos gastronómicos en cada comunidad. 


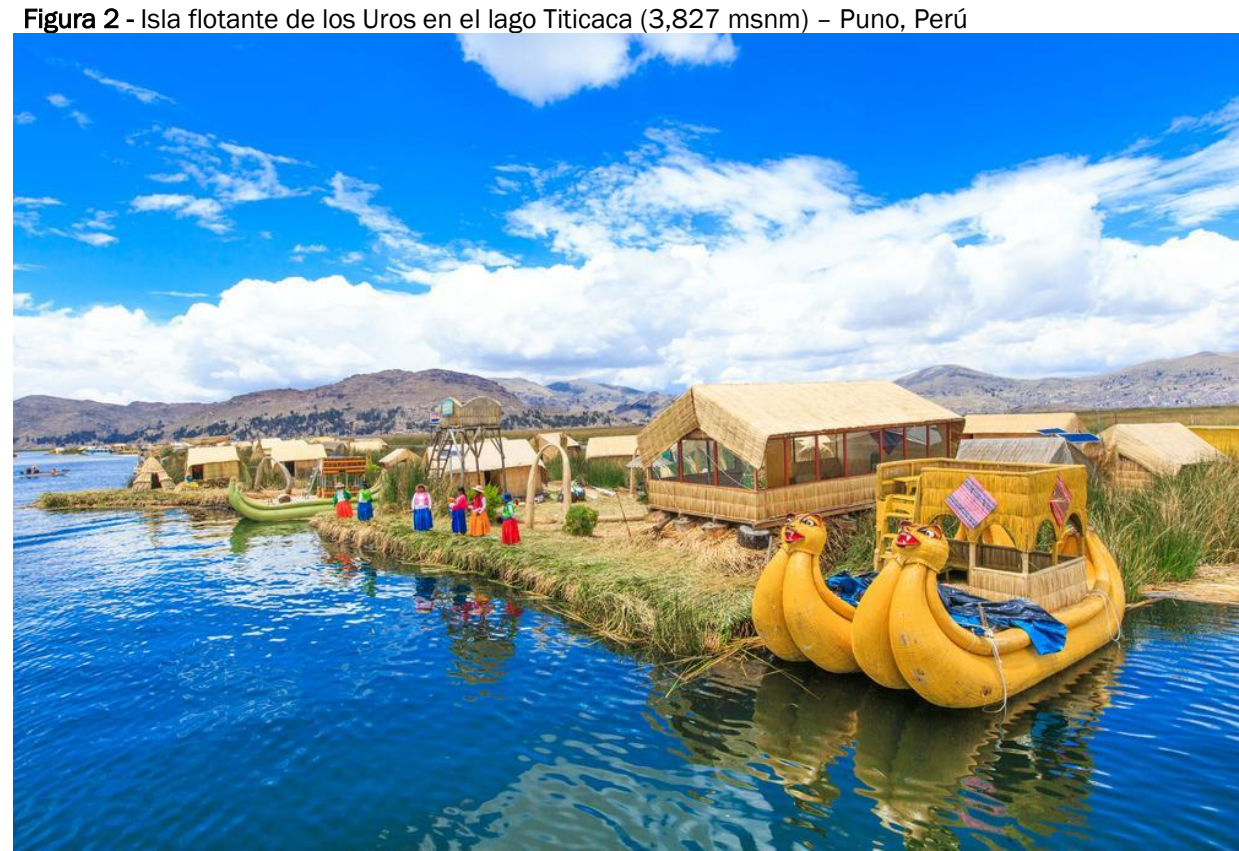

Fuente: Extraído de MiViaje (2018)

El objetivo principal de esta investigación es modelar y proyectar el número de turistas internacionales que arriban a Puno mediante un análisis de la serie histórica de los arribos de turistas internacionales y sus variaciones estacionales usando periodicidad mensual de los años 2003 a 2017. Para ello, esta investigación utiliza la metodología ARIMA (Auto-Regressive Integrated Moving Average) de Box \& Jenkins (1976) para el modelamiento y proyección de la serie estadística cuya utilidad del trabajo es principalmente la previsión en las decisiones operacionales del turismo, preparaciones de tours, infraestructura, transporte, capacitación en el servicio, entre otros. Finalmente, el documento se estructura de la siguiente manera: se presenta el marco teórico, se hace una descripción de los materiales y métodos utilizados, posteriormente se presentan los resultados utilizando la metodología de Box-Jenkins y finalmente las conclusiones más resaltantes para el presente estudio.

\section{REVISIÓN DE LITERATURA}

Al respecto, para el estudio y proyección de la demanda del turismo con series de tiempo, existen diversos trabajos de investigación, así para su modelamiento ARIMA están los trabajos de Hosking (1981), Chang et al., (2009), Loganathan \& Ibrahim (2010), Lim \& Mcaleer (1999), Peiris (2016), Reisen (1994), Nanthakumar, Subramaniam, \& Kogid (2012), Greenidge (2001). Para los costos del turismo en modelos ARIMA, el trabajo de Psillakis, Alkiviadis, \& Kanellopoulos (2009); utilizando la metodología ARIMA-GARCH están las investigaciones de Coshall (2009), Shareef \& McAleer (2005); con modelos ARMA-GARCH multivariado se cita el trabajo de Chan, Lim, \& McAleer (2005). Asimismo, trabajos que utilizan los modelos ARFIMA (modelos ARIMA Fraccionalmente Integrados) de memoria larga se encuentran los trabajos de Granger \& Joyeux (1980), Peiris \& Perera (1988), Baillie (1996); modelos ARIMA y ARFIMA que utilizan el estadístico MAPE, MAE y RMSE, están los trabajos de Chu (2008), Shitan (2008) y Lee, Song, \& Mjelde (2008); con modelos ARFIMA-FIGARCH para el turismo los trabajos de Chokethaworn et al., (2010) y Ray (1993); con modelado X-12-ARIMA y ARFIMA el trabajo de Chaitip \& Chaiboonsri (2015). Respecto del modelado de la oferta y demanda del turismo con modelos VECM el trabajo de Zhou, Bonham, \& Gangnes (2007); para la proyección de la demanda de turismo en series multivariadas y univariadas el trabajo de du Preez \& Witt (2003). Para el modelado y proyección econométrica de la demanda de turismo por MCO los trabajos de Athanasopoulos \& Hyndman (2006) y Botti, Peypoch, Randriamboarison, \& Solonandrasana (2007) y para la proyección de los ingresos debido al turismo con la metodología ARMAX el trabajo de Akal (2004). 


\section{MATERIALES Y MÉTODOS}

La selección de los materiales y métodos para la presente investigación comprende de tres partes: la descripción de los datos a utilizar, la metodología ARIMA, tests de estacionariedad y tests para elección de modelos más eficientes.

\subsection{Datos}

Para el desarrollo de esta investigación se utilizó información con período mensual para los años 2003 a 2017 extraídos de la base de datos del Banco Central de Reserva del Perú - Sucursal Puno (BCRP) para el número total de arribos de turistas internacionales al departamento de Puno.

\subsection{Metodología ARIMA estacional de Box-Jenkins}

Para la metodología se hace uso del modelo ARIMA estacional de Box \& Jenkins (1976), donde los pasos de la metodología consisten en:

Análisis preliminar: realizar un análisis preliminar a toda la información de tal modo que sea un proceso estocástico estacionario.

Identificación de un modelo tentativo: en este paso se especifica el orden $(p, d, q)$ del modelo ARIMA, para ello se hace uso de correlogramas y las funciones de autocorrelación simple y parcial.

Estimación del modelo: el siguiente paso es la estimación del modelo ARIMA identificado en el paso anterior. La estimación puede ser realizada por el método de mínimos cuadrados o máxima verosimilitud.

Diagnóstico de resultados y selección: para este paso se realiza la revisión de los modelos usando tests estadísticos para los parámetros y residuos. Asimismo, para la elección del mejor modelo se utiliza el Criterio de Informacion de Akaike (AIC) y el Criterio de Información de Schwarz (SC). Por otro lado, es posible utilizar los estadísticos Media Porcentual del Error Absoluto (MAPE), el porcentaje de medida del resultado (Z) y el coeficiente de correlación normalizado ( $r$ ) para la selección del modelo más eficiente.

Proyección: si el modelo más eficiente del paso anterior es el adecuado, entonces el modelo puede ser usado para la representación y proyección.

Para la definición del modelo ARIMA se tienen los procesos $A R(p)$ y $\mathrm{MA}(\mathrm{q})$ siguientes:

$$
\begin{aligned}
& Y_{t}=\sum_{i=1}^{p} \phi_{i} Y_{t-i}+\varepsilon_{t}, \\
& Y_{t}=\varepsilon_{t}+\sum_{i=1}^{q} \theta_{i} \varepsilon_{t-i} .
\end{aligned}
$$

Un modelo $\operatorname{ARIMA}(0, d, 0)$ es una serie temporal que se convierte en un proceso de ruido blanco después de ser diferenciada $d$ veces. El modelo $\operatorname{ARIMA}(0, d, 0)$ se expresa como $(1-L)^{d} Y_{t}=\varepsilon_{t}$ o lo que es lo mismo como $Y_{t}-Y_{t-d}=\varepsilon_{t}$. La formulación general de un modelo $\operatorname{ARIMA}(p, d, q)$ se denomina proceso integrado de medias móviles de orden $(p, d, q)$ y se escribe como

$$
Y_{t}-Y_{t-d}=\sum_{i=1}^{p} \phi_{i} Y_{t-i}+\varepsilon_{t}+\sum_{i=1}^{q} \theta_{i} \varepsilon_{t-i}
$$

O en su forma compacta,

$$
\left(1-\phi_{1} L-\phi_{2} L^{2}-\mathrm{L}-\phi_{p} L^{p}\right)(1-L)^{d} Y_{t}=\left(1-\theta_{1} L-\theta_{2} L^{2}-\mathrm{L}-\theta_{q} L^{q}\right) \varepsilon_{t}
$$


Las series con tendencia secular y variaciones cíclicas puede representarse con los modelos $A R I M A(p, d, q)(P, D, Q)$ o $\operatorname{SARIMA}(p, d, q)(P, D, Q)$. El primer paréntesis se refiere a la tendencia secular o parte regular y el segundo paréntesis a las variaciones estacionales o parte cíclica de la serie.

\subsection{Tests de estacionariedad}

\subsubsection{Prueba de raíz unitaria de Dickey-Fuller Aumentado (ADF)}

La prueba ADF de Dickey \& Fuller (1979) busca determinar la existencia de una raíz unitaria en una serie de tiempo. La hipótesis nula de esta prueba es que existe una raíz unitaria en la serie. En un modelo simple autorregresivo de orden uno, $\operatorname{AR}(1)$ :

$$
y_{t}=\rho y_{t-1}+u_{t}
$$

donde $y_{t}$ es la variable de interés, $t$ es el de tiempo, $\rho$ es un coeficiente, y $u_{t}$ es el término de error. La raíz unitaria está presente si $\rho=1$. En este caso, el modelo no sería estacionario. El modelo de regresión puede ser escrito como:

$$
\Delta y_{t}=(\rho-1) y_{t-1}+u_{t}=\delta y_{t-1}+u_{t}
$$

donde $\Delta$ es el operador de primera diferencia. Este modelo puede ser estimado y las pruebas para una raíz unitaria son equivalentes a pruebas $\delta=0$ (donde $\delta=\rho=-1$ ). Dado que la prueba se realiza con los datos residuales en lugar de los datos en bruto, no es posible utilizar una distribución estándar para proporcionar valores críticos. Por lo tanto, esta estadística tiene una determinada distribución conocida simplemente como la tabla de Dickey \& Fuller (1979).

\subsubsection{Prueba de raíz unitaria de Phillips-Perron (PP)}

La prueba PP de Phillips \& Perron (1988) es una prueba de raíz unitaria y se utiliza en el análisis de series de tiempo para probar la hipótesis nula que una serie de tiempo es integrada de orden 1. Se basa en la prueba de Dickey \& Fuller (1979) con la hipótesis nula es $\rho=0$ en $y_{t}=\rho y_{t-1}+u_{t}$ donde $\Delta$ es la primera diferencia del operador. Al igual que la prueba de Dickey-Fuller aumentada, la prueba de Phillips-Perron aborda la cuestión de que el proceso de generación de datos para $y_{t}$ podría tener un orden superior de autocorrelación que es admitido en la ecuación de prueba haciendo $y_{t-1}$ endógeno e invalidando así el Dickey-Fuller t-test. Mientras que la prueba de Dickey-Fuller aumentada aborda esta cuestión mediante la introducción de retardos de $\Delta y_{t}$ como variables independientes en la ecuación de la prueba, la prueba de Phillips-Perron hace una corrección no paramétrica a la estadística t-test.

\subsection{Tests de selección del modelo óptimo}

\subsubsection{Criterio de Información de Akaike (AIC)}

El Criterio de Informacion de Akaike fue desarrollado por Akaike (1974) y es una medida para la selección del mejor modelo estimado. En el caso general, se puede escribir la ecuación como

$$
A I C=2 k-2 \ln (L)
$$

donde $k$ es el número de parámetros en el modelo estadístico y $L$ es el valor de la función de máxima verosimilitud para el modelo estimado. 


\subsubsection{Criterio de Información de Schwarz (SC)}

El Criterio de Información de Bayes (BIC) o Criterio de Información de Schwarz (SC) fue desarrollado por Schwarz (1978) y es un criterio para elección del mejor modelo entre una clase de modelos paramétricos con diferente número de parámetros. En el caso general se escribe como

$$
-2 \ln p(x \mid k) \approx B I C=-2 \ln l
$$

donde $n$ es el número de observaciones o el tamaño muestral, $k$ el número de parámetros libres a ser estimados incluyendo la constante y $L$ el valor maximizado de la función de verosimilitud.

\subsubsection{Media Porcentual del Error Absoluto (MAPE)}

La Media Porcentual del Error Absoluto (MAPE) es una medida de la ocurrencia de una serie temporal. Esto es frecuentemente expresado como un porcentaje, la fórmula del estadístico MAPE es la siguiente:

$$
\text { MAPE }=\frac{1}{n} \sum_{i=1}^{n}\left|\frac{A_{i}-F_{i}}{A_{i}}\right|
$$

donde $A_{i}$ es el valor actual y $F_{i}$ es el valor proyectado. La diferencia entre $A_{i}$ y $F_{i}$ es dividido por el valor actual de $A_{i}$. El valor absoluto de este cálculo es sumado para cada observación proyectada en el tiempo y dividido por el número de observaciones $n$. Esto hace que sea un error porcentual, por lo que se puede comparar el error de series de tiempo ajustadas que difieren en el nivel. La interpretación para los lineamientos de MAPE es la siguiente: si el valor de MAPE es inferior al 10\% es un pronóstico "altamente preciso". Si el valor de MAPE se encuentra entre el $10 \%$ y el $20 \%$ es un pronóstico "bueno". Si el valor de MAPE se encuentra entre el 20 y el 50\% es un pronóstico "razonable". Si el valor de MAPE es superior al 50\% es un pronóstico "inexacto" (Lewis, 1982).

\subsubsection{Porcentaje de medida del resultado $(Z)$}

El valor de $Z$ es usado como una medida relativa para niveles de aceptación. Como un punto referencial para los resultados experimentales óptimos, $Z$ se utilizará a un valor de $\pm 5 \%$, de este modo se define el estadístico como:

$$
Z=\frac{\sum_{i=1}^{n} j}{n} * 100 \% \text { para } \begin{cases}j=1 & \text { si }\left|\frac{A_{i}-F_{i}}{A_{i}}\right| \\ j=0 & \text { si } \text { otro caso }\end{cases}
$$

donde $A_{i}$ es el valor actual y $F_{i}$ es el valor proyectado y $n$ el número de observaciones utilizadas. Para la elección del mejor modelo se debe considerar aquel que cuente con un valor $Z$ mayor.

\subsubsection{Coeficiente de correlación normalizado $(r)$}

El coeficiente de correlación normalizado $r$ es una medida de la cercanía de las observaciones y su proyección, se define como:

$$
r=\frac{\sum_{i=1}^{n} A_{i}^{*} F_{i}}{\sqrt{\sum_{i=1}^{n}\left(A_{i}\right)^{2} * \sum_{i=1}^{n}\left(F_{i}\right)^{2}}}
$$

donde $A_{i}$ es el valor actual y $F_{i}$ es el valor proyectado. Para realizar la elección del mejor modelo se debe elegir aquel que cuente con el estadístico $r$ mayor. 


\section{RESULTADOS}

Para la presentación de los resultados, se utiliza la metodología ARIMA de Box \& Jenkins (1976) que consta de la identificación, estimación del modelo, examen de diagnóstico y finalmente la proyección de la serie. Para el análisis de los datos se utilizó el software estadístico Eviews 9, donde se hace uso de un total de 177 arribos de turistas internacionales con un mínimo de 4,650 arribos y un máximo de 36,147 descritos en la Tabla 1.

Tabla 1 - Estadística descriptiva

\begin{tabular}{llccccc}
\hline Lista de variables & Abreviatura & Obs. & Media & $\begin{array}{l}\text { Desv. } \\
\text { Estánd. }\end{array}$ & Mínimo & Máximo \\
\hline Arribo de turistas internacionales a Puno & arribos & 177 & 18,809 & 8,341 & 4,650 & 36,147 \\
\hline
\end{tabular}

Fuente: Elaborado por los autores

Para la identificación, la Figura 3 muestra la evolución de los arribos de turistas internacionales al departamento de Puno para los años 2003 a 2017, mostrando claramente un crecimiento y da evidencia a la presencia de no estacionariedad en media y varianza.

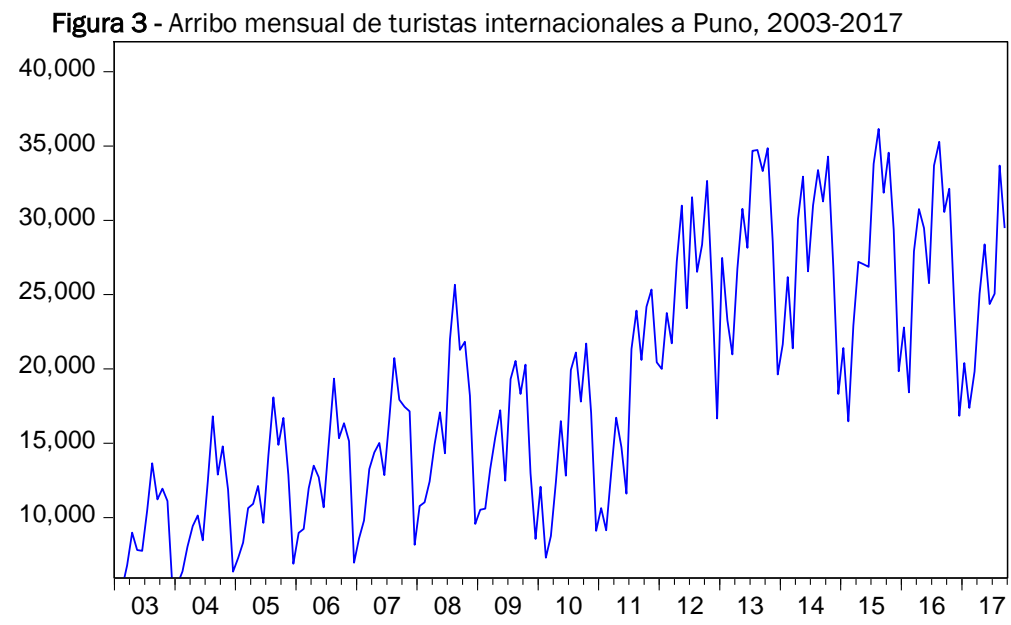

Fuente: Elaborado por los autores

En la Figura 4 muestra que cada año los arribos de turistas a Puno presentan un ciclo estacional anual debido que empiezan a subir desde el mes de febrero hasta mayo, cayendo levemente en el mes de junio recuperándose en julio y llegando a su máximo en agosto cayendo en setiembre recuperándose levemente en octubre y cayendo al máximo en el mes de diciembre. Lo que da evidencia para un modelo estacional ARIMA de 12 meses.

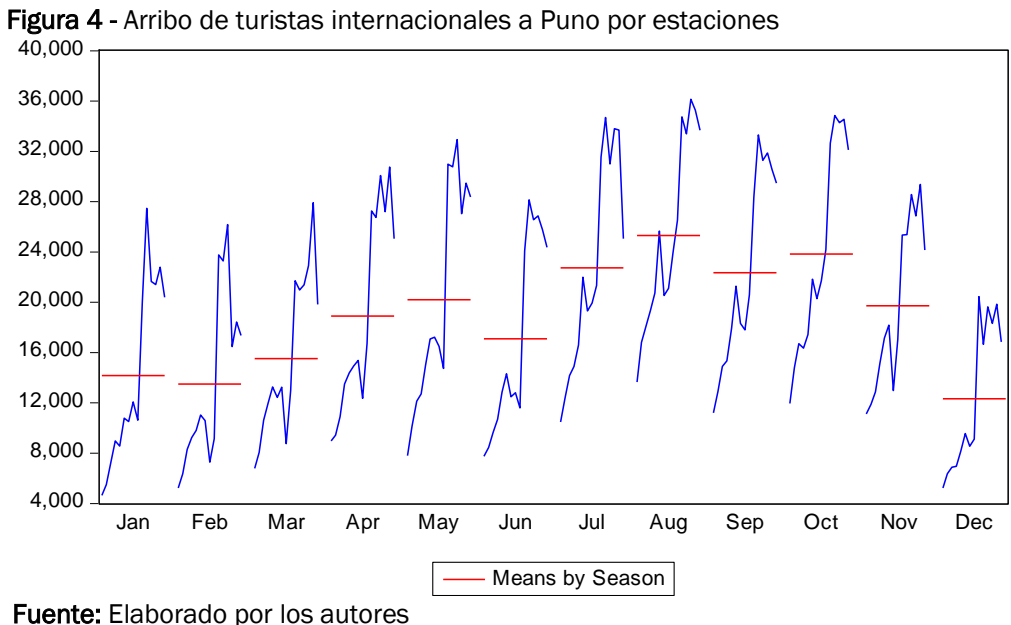




\subsection{Tests de estacionariedad}

Como primer paso se determina si la serie es estacionaria, para ello se utiliza las pruebas de raíz unitaria ADF de Dickey \& Fuller (1979), PP de Phillips \& Perron (1988) y KPSS de Kwiatkowski, Phillips, Schmidt, \& Shin (1992) las que se muestran en la Tabla 2.

\begin{tabular}{|c|c|c|c|c|c|c|}
\hline \multirow[b]{2}{*}{ Variable } & \multicolumn{2}{|l|}{ Ninguno } & \multicolumn{2}{|c|}{ Con intercepto } & \multicolumn{2}{|c|}{ Con intercepto y tendencia } \\
\hline & Nivel & $\begin{array}{l}\text { Primera dife- } \\
\text { rencia }\end{array}$ & Nivel & $\begin{array}{l}\text { Primera dife- } \\
\text { rencia }\end{array}$ & Nivel & $\begin{array}{l}\text { Primera dife- } \\
\text { rencia }\end{array}$ \\
\hline Test de ADF & $\begin{array}{l}0.716 \\
(0.868)\end{array}$ & $\begin{array}{l}-2.68 * * \\
(0.007)\end{array}$ & $\begin{array}{l}-1.335 \\
(0.612)\end{array}$ & $\begin{array}{l}-2.7901 \\
(0.061)\end{array}$ & $\begin{array}{l}-1.577 \\
(0.798)\end{array}$ & $\begin{array}{l}-2.868 \\
(0.175)\end{array}$ \\
\hline Test de PP & $\begin{array}{l}-0.158 \\
(0.627)\end{array}$ & $\begin{array}{l}-19.742 * * \\
(0.000)\end{array}$ & $\begin{array}{l}-3.345 * \\
(0.014)\end{array}$ & $\begin{array}{l}-20.061 * * \\
(0.000)\end{array}$ & $\begin{array}{l}-6.313 * * \\
(0.000)\end{array}$ & $\begin{array}{l}-20.003 * * \\
(0.000)\end{array}$ \\
\hline Test de KPSS & - & $\begin{array}{l}- \\
--\end{array}$ & $\begin{array}{l}1.536 \\
(0.463) \sim\end{array}$ & $\begin{array}{l}0.045 * * \\
(0.463) \sim\end{array}$ & $\begin{array}{l}0.112^{*} \\
(0.146) \sim\end{array}$ & $\begin{array}{l}0.041^{*} \\
(0.146) \sim\end{array}$ \\
\hline
\end{tabular}

Notas: $\left({ }^{*}\right)$ y $\left({ }^{* *}\right)$ denota significancia estadística al $5 \%$ y $1 \%$, respectivamente. Valores en () indican el p-value de Mackinnon (1996). El símbolo ( ) indica el valor crítico asintótico de Kwiatkowski et al., (1992)

Fuente: Elaborado por los autores

En la Tabla 2 se muestra la realización de tres diferentes test de estacionariedad a un nivel de $1 \%$ y $5 \%$ de nivel de significancia y se concluye que los arribos de turistas internacionales no es estacionaria en niveles al $1 \%$ de significancia. Para este propósito se calculó la serie en primera diferencia y dando como resultado que para los test de PP y KPSS la serie es estacionaria a un $1 \%$ de significancia, lo que indica que la serie es estacionaria en primera diferencia.

\subsection{Modelos autoregresivos y de medias móviles}

Los datos en logaritmos de los arribos de turistas internacionales a Puno son usados para modelar el turismo. En la Tabla 3 se presenta estimaciones de cuatro modelos autoregresivos (AR), medias móviles (MA) y modelos autoregresivos integrados y de medias móviles (ARIMA) que previamente se revisó los correlogramas para la verificación y su estacionalidad, es así que se estimó por la metodología de mínimos cuadrados para determinar el comportamiento de los arribos de turistas internacionales a Puno durante 2003:m1 a 2017:m9. Asimismo, se calculó el Criterio de Información de Akaike (AIC), Criterio de Información de Schwarz (SC) para la elección del mejor modelo y el estadístico de Durwin-Watson (DW) para un primer análisis de presencia de autocorrelación en los modelos estimados.

Para la selección del modelo, la Tabla 3 se muestra que los modelos con mayor ajuste no presentan problemas de autocorrelación, debido que el estadístico de Durbin-Watson (DW) de los modelos se encuentran alrededor de 2 (Durbin \& Watson, 1950, 1971). Utilizando el Criterio de Información de Akaike (AIC) debido a Akaike (1974) y Criterio de Información de Schwarz (SC) a Schwarz (1978) para la elección del mejor modelo, de la Tabla 3 se tiene que el mejor modelo que presenta los estadísticos mínimos de AIC y SC es el Modelo 1 dado bajo su especificación como SARIMA $(6,1,24)(1,0,1) 12$ es el mejor modelo para representar a los arribos de turistas internacionales a la región de Puno para los períodos 2003 a 2017.

Asimismo, para evaluar la eficiencia de los modelos ARIMA de la Tabla 3, se construyó los estadísticos MAPE, $Z$ y $r$ que se muestra a continuación: 
Tabla 3 - Estimación de modelos ARIMA para arribo de turistas internacionales a Puno

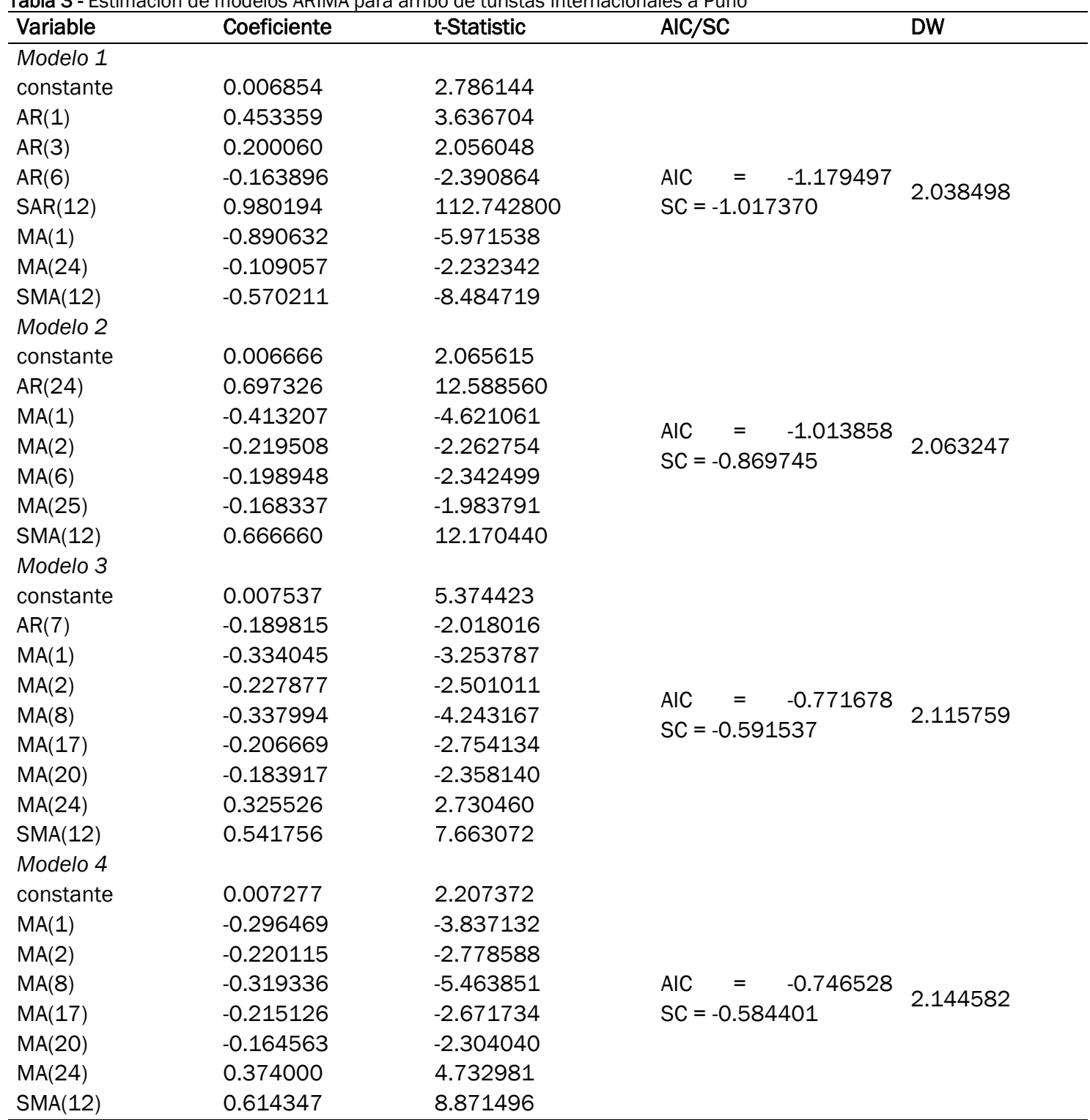

Notas: AIC y SC son el Criterio de Información de Akaike y Criterio de Schwarz, respectivamente. DW se refiere al estadístico Durbin-Watson de autocorrelación

Fuente: Elaborado por los autores

\subsubsection{Estadístico MAPE}

La Media Porcentual del Error Absoluto (MAPE) es una medida de la ocurrencia de una serie temporal. Esto es frecuentemente expresado como un porcentaje, la fórmula del estadístico MAPE es la siguiente (Lewis, 1982):

$$
M A P E=\frac{1}{n} \sum_{i=1}^{n}\left|\frac{A_{i}-F_{i}}{A_{i}}\right|
$$

donde $A_{i}$ es el valor actual y $F_{i}$ es el valor proyectado. La diferencia entre $A_{i}$ y $F_{i}$ es dividido por el valor actual de $A_{i}$. El valor absoluto de este cálculo es sumado para cada observación proyectada en el tiempo y dividido por el número de observaciones $n$ proyectadas en el tiempo. Esto hace que sea un error porcentual, por lo que se puede comparar el error de series de tiempo ajustadas que difieren en el nivel. Y también este papel utiliza la medida de precisión MAPE. Los lineamientos para MAPE, la interpretación es la siguiente: si el valor de MAPE es inferior al 10\%, es un pronóstico "altamente preciso". Si el valor de MAPE se encuentra 
entre el $10 \%$ y el $20 \%$, es un pronóstico "bueno". Si el valor de MAPE se encuentra entre el $20 \%$ y el $50 \%$, es un pronóstico "razonable". Si el valor de MAPE es superior al 50\%, es un pronóstico "inexacto" (Lewis, 1982).

Para la construcción de MAPE en este trabajo se utiliza la proyección del paso anterior para cada uno de los mejores modelos planteados. Para realizar el cálculo para cada una de las proyecciones se utilizó la siguiente fórmula

$$
\text { MAPE }=\frac{1}{n} \sum_{i=1}^{n}\left|\frac{\text { arribos }_{i}-\text { arribosfmJ }_{i}}{\text { arribos }_{i}}\right|, \quad J=1,2,3,4
$$

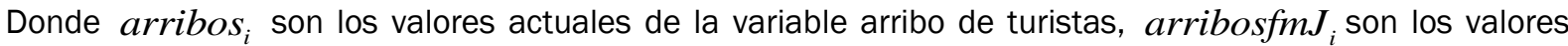
proyectados de la variable arribo de turistas utilizando los modelos ARIMA en $J=1,2,3$ y 4 . Los resultados del cálculo se muestran en la Tabla 4.

\subsubsection{Porcentaje de medida de resultado $(Z)$}

El valor de $Z$ es usado como una medida relativa para niveles de aceptación. Como un punto referencial para los resultados experimentales óptimos, $Z$ se utiliza a un valor de $\pm 5 \%$ (Law \& Au, 1999), de este modo se define el estadístico como:

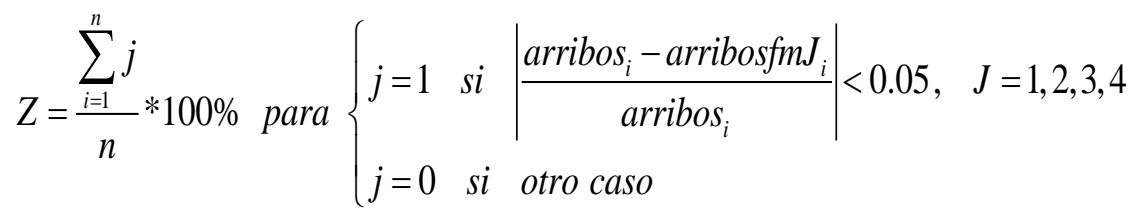

donde arribos $_{i}$ son los valores actuales de la variable arribo de turistas, arribosfmJ ${ }_{i}$ son los valores proyectados de la variable arribo de turistas utilizando los modelos ARIMA $J=1,2$, 3 y 4, los resultados del cálculo se muestran en la Tabla 4.

\subsubsection{Coeficiente de correlación normalizado $(r)$}

El coeficiente de correlación normalizado $r$ es una medida de la cercanía de las observaciones y su proyección (Law \& Au, 1999), se define como:

$$
r=\frac{\sum_{i=1}^{n} \text { arribos }_{i} \text { arribosfmJ }_{i}}{\sqrt{\sum_{i=1}^{n}\left(\text { arribos }_{i}\right)^{2} * \sum_{i=1}^{n}\left(\text { arribosfmJ }_{i}\right)^{2}}}, J=1,2,3,4
$$

\begin{tabular}{|c|c|c|c|c|}
\hline Modelos & & MAPE & $\mathbf{Z}$ & $r$ \\
\hline Modelo 1 & SARIMA $(6,1,24)(1,0,1)_{12}$ & 16.15 & 16.45 & 0.9836 \\
\hline Modelo 2 & SARIMA $(24,1,25)(0,0,1) 12$ & 19.01 & 15.13 & 0.9781 \\
\hline Modelo 3 & $\operatorname{SARIMA}(7,1,24)(0,0,1)_{12}$ & 25.30 & 7.69 & 0.9668 \\
\hline Modelo 4 & $\operatorname{SARIMA}(0,1,24)(0,0,1)_{12}$ & 45.36 & 2.27 & 0.9665 \\
\hline
\end{tabular}

donde arribos $_{i}$ son los valores actuales de la variable arribo de turistas, arribosfmJ ${ }_{i}$ son los valores proyectados de la variable arribo de turistas internacionales utilizando los modelos ARIMA $J=1,2$, 3 y 4 .

Fuente: Elaborado por los autores

La Tabla 4 muestra los estadísticos MAPE, porcentaje de medida de resultado (Z) y coeficiente de correlación normalizado ( $r$ ) para la elección del mejor modelo planteado. De los resultados se tiene que el Modelo 1 cuya especificación es SARIMA $(6,1,24)(1,0,1) 12$ es el modelo más adecuado debido que presenta el menor valor del estadístico MAPE igual a $16.15 \%$. 
Figura 5 - Raíces inversas de los polinomios AR/MA de SARIMA $(6,1,24)(1,0,1)_{12}$

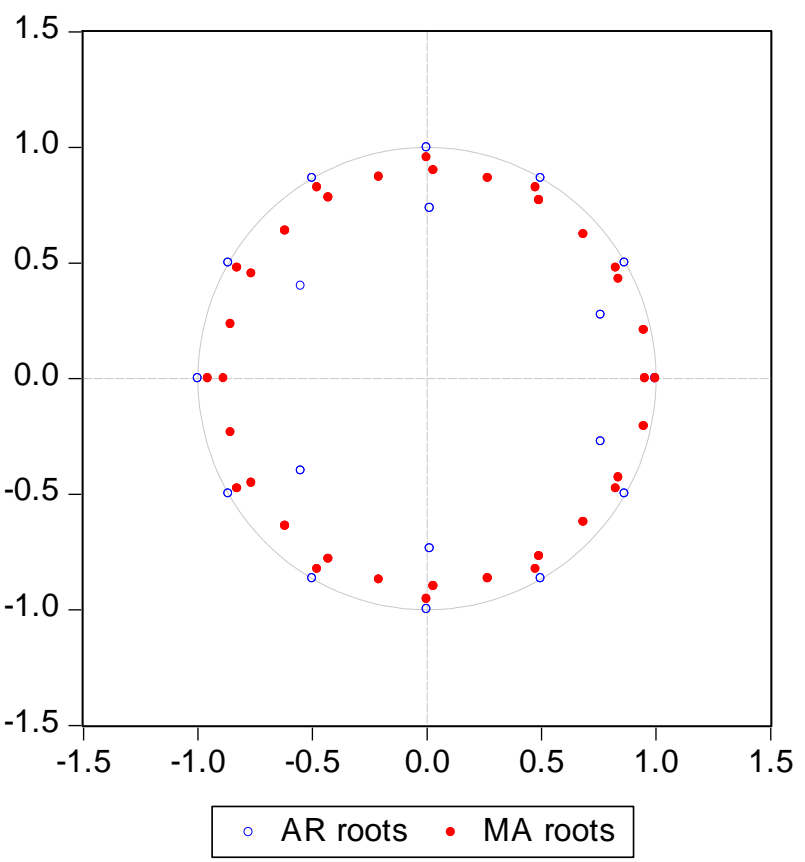

\begin{tabular}{lll}
\hline \hline \multicolumn{1}{c}{ AR Root(s) } & Modulus & Cycle \\
\hline \hline$-0.864583 \pm 0.499167 \mathrm{i}$ & 0.998334 & 2.400000 \\
-0.998334 & 0.998334 & \\
$-5.55 \mathrm{e}-17 \pm 0.998334 \mathrm{i}$ & 0.998334 & 4.000000 \\
$0.499167 \pm 0.864583 \mathrm{i}$ & 0.998334 & 6.000000 \\
$0.864583 \pm 0.499167 \mathrm{i}$ & 0.998334 & 12.00000 \\
0.998334 & 0.998334 & \\
$-0.499167 \pm 0.864583 \mathrm{i}$ & 0.998334 & 3.000000 \\
$0.761295 \pm 0.273329 \mathrm{i}$ & 0.808875 & 18.22811 \\
$0.013951 \pm 0.736797 \mathrm{i}$ & 0.736929 & 4.048800 \\
$-0.548567 \pm 0.400430 \mathrm{i}$ & 0.679168 & 2.502221 \\
\hline \hline
\end{tabular}

No root lies outside the unit circle. ARMA model is stationary.

\begin{tabular}{lcc}
\hline \multicolumn{1}{c}{ MA Root(s) } & Modulus & Cycle \\
\hline \hline 0.999911 & 0.999911 & \\
$0.947992 \pm 0.208563 \mathrm{i}$ & 0.970664 & 29.01420 \\
$0.477133 \pm 0.826419 \mathrm{i}$ & 0.954266 & 6.000000 \\
$-2.78 \mathrm{e}-16 \pm 0.954266 \mathrm{i}$ & 0.954266 & 4.000000 \\
$-0.826419 \pm 0.477133 \mathrm{i}$ & 0.954266 & 2.400000 \\
0.954266 & 0.954266 & \\
$0.826419 \pm 0.477133 \mathrm{i}$ & 0.954266 & 12.00000 \\
-0.954266 & 0.954266 & \\
$-0.477133 \pm 0.826419 \mathrm{i}$ & 0.954266 & 3.000000 \\
$0.838561 \pm 0.428936 \mathrm{i}$ & 0.941898 & 13.28886 \\
$0.684739 \pm 0.621909 \mathrm{i}$ & 0.925008 & 8.521306 \\
$0.490908 \pm 0.770682 \mathrm{i}$ & 0.913751 & 6.260463 \\
$0.267901 \pm 0.865104 \mathrm{i}$ & 0.905635 & 4.945491 \\
$0.029861 \pm 0.899055 \mathrm{i}$ & 0.899551 & 4.086374 \\
$-0.207532 \pm 0.870530 \mathrm{i}$ & 0.894926 & 3.481326 \\
$-0.428415 \pm 0.781736 \mathrm{i}$ & 0.891432 & 3.032242 \\
$-0.617923 \pm 0.638951 \mathrm{i}$ & 0.888868 & 2.685735 \\
$-0.763262 \pm 0.452104 \mathrm{i}$ & 0.887111 & 2.410278 \\
$-0.854598 \pm 0.234112 \mathrm{i}$ & 0.886084 & 2.186058 \\
-0.885747 & 0.885747 & \\
\hline \hline
\end{tabular}

No root lies outside the unit circle.

ARMA model is invertible.

Fuente: Elaboración propia 
Asimismo, el Modelo 1 presenta el mayor valor del porcentaje de medida de resultado (Z) igual a 16.45 y el mayor valor del coeficiente de correlación normalizado $r=0.9836$. Luego, se concluye que el Modelo 1 es el mejor modelo debido que presenta los menores valores de los Criterio de Información de Akaike (AIC) y del Criterio de Schwarz (SC) del paso anterior y asimismo presenta el menor valor de MAPE, mayor valor $Z$ y $r$, luego el Modelo 1 cuya especificación es $\operatorname{SARIMA}(6,1,24)(1,0,1) 12$ se puede utilizar para la representación de la demanda de turismo en la región de Puno y su proyección.

\section{DIAGNÓSTICO AL MODELO SARIMA $(6,1,24)(1,0,1)_{12}$ ELEGIDO}

Para el diagnóstico del modelo SARIMA $(6,1,24)(1,0,1) 12$ la Figura 5 muestra que las raíces de todos los AR y MA son menores de 1, esto muestra que el modelo ARIMA es estable al igual que los errores. Asimismo, la Figura 6 muestra los valores actuales, los valores proyectados y los residuos del modelo SARIMA (6, 1, $24)(1,0,1)^{12}$.

Incorrelación. En la Figura 7, se muestra el correlograma del modelo SARIMA (6, 1, 24)(1, 0, 1)12 analizada por el estadístico Q de Ljung-Box (Ljung \& Box, 1978), determina que hay ausencia de autocorrelación en los residuos, es decir el comportamiento se asemeja al de un ruido blanco. Se observa también que todos los coeficientes caen dentro de la banda de confianza al 95\% de confianza, además todos los p-valores asociados al estadístico de Ljung-Box para cada retardo ( $p$-value) son lo suficientemente grandes como para no rechazar la hipótesis nula que todos los coeficientes son nulos. Asimismo de la Tabla 3 se muestra que el modelo SARIMA $(6,1,24)(1,0,1)_{12}$, Modelo 1, no presenta problemas de autocorrelación, debido que el estadístico de Durbin-Watson (DW) se encuentra alrededor de 2 (Durbin \& Watson, 1950, 1971). En consecuencia los residuos del modelo $\operatorname{SARIMA}(6,1,24)(1,0,1) 12$ se encuentran no correlacionados.

Figura 6 - Residuos del modelo SARIMA $(6,1,24)(1,0,1)_{12}$

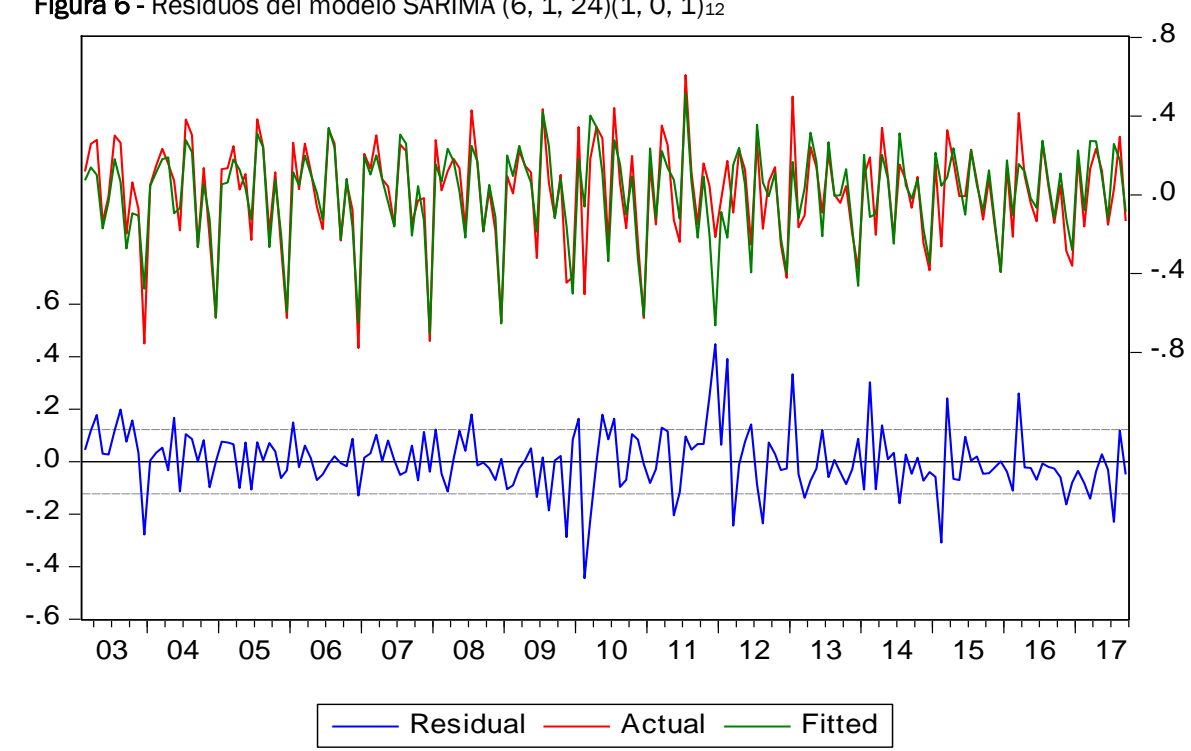

Fuente: Elaborado por los autores 
Figura 7 - Correlograma de los residuos del modelo SARIMA $(6,1,24)(1,0,1)_{12}$

\begin{tabular}{|c|c|c|c|c|c|c|}
\hline Autocorrelation & Partial Correlation & & $A C$ & PAC & Q-Stat & Prob \\
\hline 111 & 111 & 1 & -0.022 & -0.022 & 0.0839 & \\
\hline 111 & 101 & 2 & 0.021 & 0.020 & 0.1619 & \\
\hline 111 & 11 & 3 & 0.019 & 0.020 & 0.2273 & \\
\hline 1 & יל 1 & 4 & 0.080 & 0.080 & 1.3895 & \\
\hline 101 & 1 1 & 5 & 0.038 & 0.041 & 1.6579 & \\
\hline 111 & $1 \mid 1$ & 6 & 0.025 & 0.023 & 1.7688 & \\
\hline 111 & 111 & 7 & 0.020 & 0.017 & 1.8445 & \\
\hline 101 & 101 & 8 & -0.043 & -0.051 & 2.1823 & 0.140 \\
\hline 1]1 & 101 & 9 & -0.017 & -0.028 & 2.2365 & 0.327 \\
\hline 101 & $1 \mid 1$ & 10 & 0.026 & 0.020 & 2.3618 & 0.501 \\
\hline $\begin{array}{lll}1 & 1\end{array}$ & $\begin{array}{lll}1 & 1\end{array}$ & 11 & 0.001 & 0.000 & 2.3621 & 0.669 \\
\hline 111 & \begin{tabular}{l|l}
1 & 1
\end{tabular} & 12 & -0.014 & -0.008 & 2.3976 & 0.792 \\
\hline וh1 & 1 1 & 13 & 0.055 & 0.061 & 2.9792 & 0.811 \\
\hline ום 1 & ים 1 & 14 & 0.090 & 0.095 & 4.5518 & 0.714 \\
\hline ו 1 & 1 & 15 & 0.067 & 0.073 & 5.4202 & 0.712 \\
\hline 101 & 101 & 16 & -0.026 & -0.028 & 5.5535 & 0.784 \\
\hline 1]1 & 101 & 17 & -0.024 & -0.045 & 5.6666 & 0.842 \\
\hline ום 1 & 1 1 1 & 18 & 0.091 & 0.070 & 7.3064 & 0.774 \\
\hline 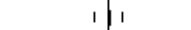 & $1[1$ & 19 & 0.018 & 0.005 & 7.3738 & 0.832 \\
\hline C. & C. & 20 & -0.119 & -0.136 & 10.224 & 0.676 \\
\hline 回 & ב & 21 & -0.102 & -0.116 & 12.334 & 0.580 \\
\hline 101 & 101 & 22 & -0.051 & -0.055 & 12.854 & 0.614 \\
\hline 1 1 & ולי & 23 & 0.040 & 0.054 & 13.188 & 0.659 \\
\hline \begin{tabular}{l|l}
1 & 1
\end{tabular} & 110 & 24 & 0.007 & 0.035 & 13.199 & 0.723 \\
\hline 101 & 101 & 25 & -0.067 & -0.051 & 14.135 & 0.720 \\
\hline ים 1 & ין ו & 26 & 0.077 & 0.109 & 15.374 & 0.699 \\
\hline 10 & $1[1$ & 27 & -0.097 & -0.080 & 17.345 & 0.630 \\
\hline 1 1 & \begin{tabular}{l|l}
1 & 1
\end{tabular} & 28 & 0.035 & -0.005 & 17.608 & 0.674 \\
\hline 1 & 1 & 29 & 0.052 & 0.034 & 18.182 & 0.695 \\
\hline 1 1 1 & 191 & 30 & 0.075 & 0.073 & 19.400 & 0.678 \\
\hline 101 & 101 & 31 & -0.067 & -0.055 & 20.358 & 0.676 \\
\hline \begin{tabular}{l|l}
1 & 1
\end{tabular} & 101 & 32 & -0.008 & -0.035 & 20.371 & 0.727 \\
\hline 111 & 101 & 33 & -0.011 & -0.035 & 20.397 & 0.772 \\
\hline 叫 1 & 101 & 34 & -0.091 & -0.062 & 22.209 & 0.727 \\
\hline C. & 101 & 35 & -0.115 & -0.081 & 25.153 & 0.619 \\
\hline 1디 1 & 叫 1 & 36 & -0.095 & -0.111 & 27.174 & 0.562 \\
\hline
\end{tabular}

Fuente: Elaborado por los autores

Normalidad. El estadístico de Jarque-Bera desarrollado por Jarque \& Bera $(1980,1981,1987)$ es una prueba de bondad de ajuste para verificar si los datos de estudios tienen asimetría o curtosis en una distribución normal, es decir si los residuos se comportan como una función normal. En la Figura 8, se muestra los resultados de este estadístico, en este caso el valor de la probabilidad igual a cero indica el rechazo de la hipótesis de una distribución normal. Como el valor del estadístico de Jarque-Bera es superior al valor de referencia de tablas (aproximadamente un valor de 6) y la probabilidad es menor a $\alpha=5 \%$, los residuos del modelo no se compartan como una función normal. Sin embargo, siguiendo al teorema central del límite, se puede concluir que al trabajar muestras más grandes que la actual, garantizaría que los errores se comporten como una función asintóticamente normal (Laurente \& Poma, 2016).

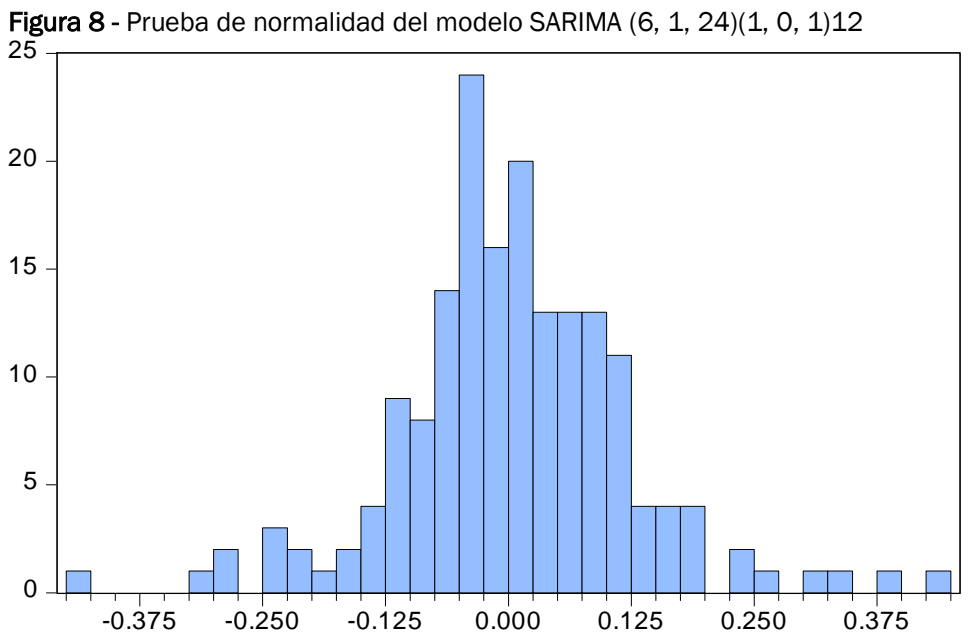

\begin{tabular}{|lc|}
\hline \multicolumn{2}{|l|}{ Series: Residuals } \\
Sample 2003M02 2017M09 \\
Observations & 176 \\
Mean & 0.004626 \\
Median & 0.000488 \\
Maximum & 0.447672 \\
Minimum & -0.444267 \\
Std. Dev. & 0.119719 \\
Skewness & 0.132088 \\
Kurtosis & 5.228002 \\
& \\
Jarque-Bera & 36.91438 \\
Probability & 0.000000 \\
\hline
\end{tabular}

Fuente: Elaborado por los autores 


\subsection{Análisis de intervención al modelo SARIMA $(6,1,24)(1,0,1)_{12}$ elegido}

En la Figura 7 del modelo $\operatorname{SARIMA}(6,1,24)(1,0,1) 12$, se comprobó que los residuos siguen un comportamiento de ruido blanco, es decir los residuos son incorrelacionados. Por otro lado, revisando la Figura 3 y la gráfica de error se verifica la existencia de dos quiebres estructurales (Chow, 1960) dadas en febrero de 2010 (2010m2) y en febrero de 2012 (2012m2) que básicamente se debió a la crisis internacional de 2008 que afecto a la visita de turistas internacionales a Puno. La Tabla 7 muestra la estimación del modelo SARIMA $(6,1,24)(1,0,1) 12$ con intervención en ambas fechas que representa una variable dummy con valor 1 para la fecha indicada y 0 para su complementario, ambas variables se representan con el nombre de D2010m2 para la variable dummy de intervención en el mes de febrero de 2010 y la variable dummy D2012m2 para la intervención en el mes de febrero de 2012.

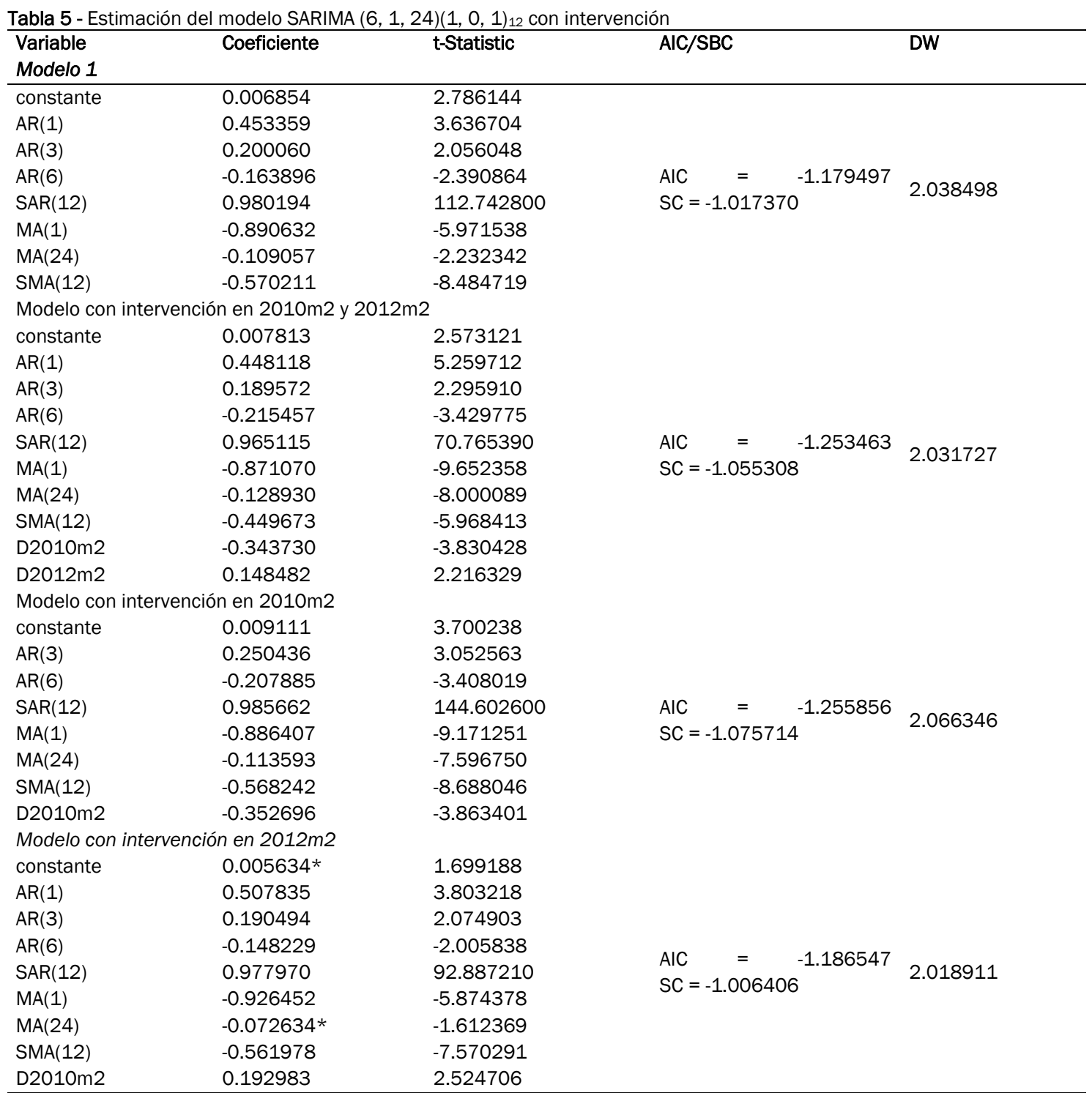

Notas: ${ }^{*}$ ) significa no significativo al 5\%. AIC y SC son el Criterio de Información de Akaike y Criterio de Schwarz, respectivamente. DW se refiere al estadístico Durbin-Watson de autocorrelación

Fuente: Elaborado por los autores

De los resultados de la Tabla 7 se muestra la estimación del modelo SARIMA $(6,1,24)(1,0,1)_{12}$ elegido previamente y tres modelos que añaden al modelo indicado la intervención en los períodos $2010 \mathrm{~m} 2$ y 2012m2, dando como resultado que el mejor modelo ARIMA con intervención, es el modelo con intervención 
en 2010m2 donde la variable que la representa D2010m2 es estadísticamente significativa y donde el modelo presenta el menor valor de los estadísticos AIC (-1.255856) y SC (-1.075714) y con un valor de $\mathrm{DW}=2.066346$ muy próximo a 2, lo que evidenciaría la ausencia de autocorrelación en este modelo. Asimismo, este modelo SARIMA $(6,1,24)(1,0,1) 12$ con intervención en 2010m2 presenta los estadísticos de AIC y SC más pequeños que el Modelo 1 debido que se está corrigiendo el quiebre en ese período.

Figura 9 - Correlograma de los residuos del modelo SARIMA $(6,1,24)(1,0,1)_{12}$ con intervención en 2010m2

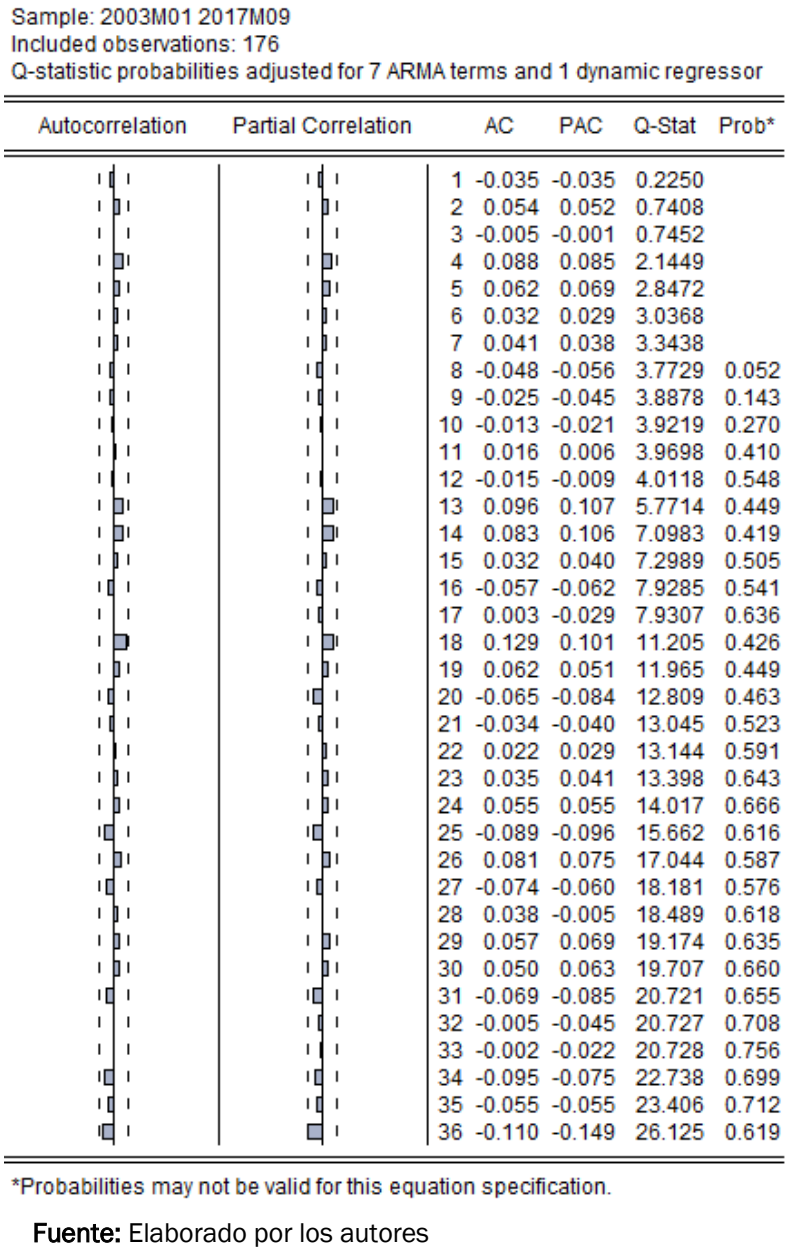

En la Figura 9, se muestra el correlograma de los residuos del modelo SARIMA $(6,1,24)(1,0,1)_{12}$ con intervención en 2010m2 analizada por el estadístico Q de Ljung-Box (Ljung \& Box, 1978), determina que hay ausencia de autocorrelación en los residuos, es decir el comportamiento se asemeja al de un ruido blanco debido que todos los coeficientes caen dentro de la banda de confianza al 95\% de confianza, además todos los p-valores asociados al estadístico de Ljung-Box para cada retardo ( $p$-value) son lo suficientemente grandes como para no rechazar la hipótesis nula que todos los coeficientes son nulos.

Asimismo, de la Tabla 5 se muestra que el modelo SARIMA (6, 1, 24)(1, 0, 1)12 con intervención en 2010m2, no presenta problemas de autocorrelación ya que el estadístico de Durbin-Watson (DW) se encuentra alrededor de 2. En consecuencia los residuos del modelo $\operatorname{SARIMA}(6,1,24)(1,0,1) 12$ con intervención en 2010m2 se encuentran no correlacionados. 
Figura 10 - Prueba de normalidad del modelo SARIMA $(6,1,24)(1,0,1)_{12}$ con intervención en $2010 \mathrm{~m} 2$

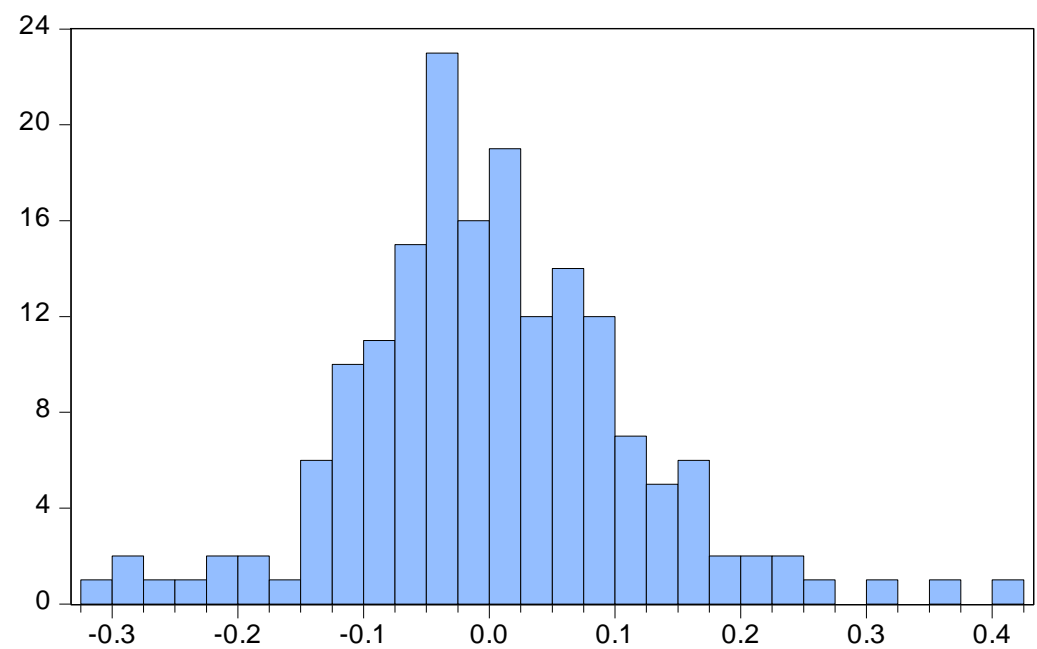

\begin{tabular}{|c|c|}
\hline Median & -0.004196 \\
\hline Maximum & 0.424600 \\
\hline Minimum & -0.310731 \\
\hline Std. Dev. & 0.113468 \\
\hline Skewness & 0.369577 \\
\hline Kurtosis & 4.410573 \\
\hline Jarq & 18.5 \\
\hline Probability & 0.000 \\
\hline
\end{tabular}

Fuente: Elaborado por los autores

Para verificar si los residuos del modelo SARIMA $(6,1,24)(1,0,1) 12$ con intervención en $2010 \mathrm{~m} 2$ se comportan como una distribución normal, la Figura 10 muestra el histograma para el estadístico de JarqueBera donde su valor de probabilidad es igual a cero, lo que indica el rechazo de la hipótesis de una distribución normal de los errores ya que el valor del estadístico de Jarque-Bera es superior al valor de referencia de tablas (aproximadamente un valor de 6) y la probabilidad es menor a $\alpha=5 \%$. Sin embargo, siguiendo al teorema central del límite, al trabajar con muestras más grandes se garantiza que los residuos se comporten como una función normal (Laurente \& Poma, 2016).

\subsection{Proyección utilizando el modelo SARIMA $(6,1,24)(1,0,1)_{12}$ elegido}

Después del examen de diagnóstico realizado al modelo SARIMA $(6,1,24)(1,0,1) 12$, se realiza la proyección de la variable de estudio (Box \& Jenkins, 1976). Los resultados se muestran en la Figura 11 donde la variable arribos es la variable original, arribosf es la proyección con el modelo ARIMA seleccionado y la variable arribosf2010m2 es la proyección con el modelo ARIMA seleccionado con intervención en $2010 \mathrm{~m} 2$.

Figura 11 - Información actual y proyectada con modelo SARIMA $(6,1,24)(1,0,1)_{12}$

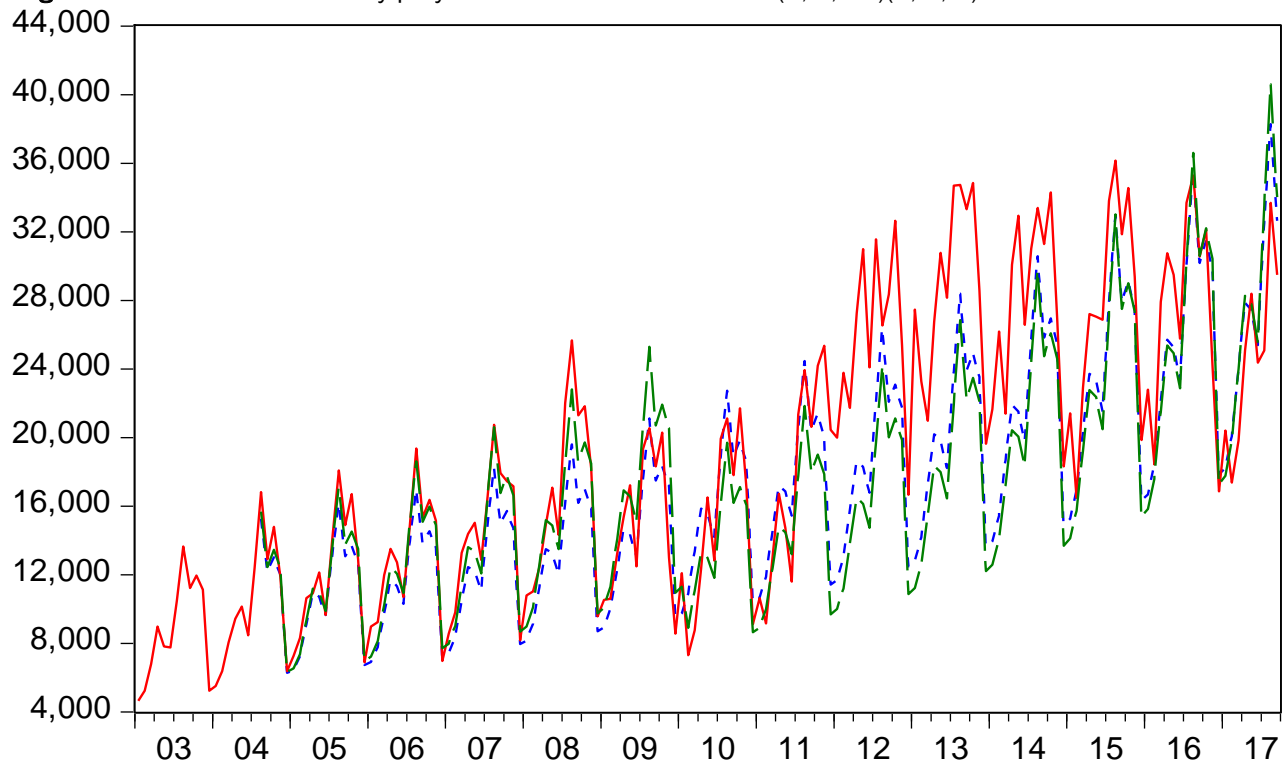

$$
\text { - - - ARRIBOSF - ARRIBOS - ARRIBOSF2010M2 }
$$

Fuente: Elaborado por los autores 
Finalmente, haciendo uso del modelo $\operatorname{SARIMA}(6,1,24)(1,0,1)_{12}$ se presenta la proyección a dos años de los arribos de turistas internacionales a la región Puno, la misma que es de utilizada para la administración en este sector.

Tabla 6 - Proyección de la demanda de turismo utilizando SARIMA $(6,1,24)(1,0,1)_{12}$

\begin{tabular}{|c|c|c|c|c|c|c|}
\hline \multirow{2}{*}{ Mes año } & \multicolumn{3}{|c|}{ Sin intervención } & \multicolumn{3}{|c|}{ Con intervención en $2010 \mathrm{~m} 2$} \\
\hline & turistas & inferior & superior & turistas & inferior & superior \\
\hline Octubre 2017 & 31,028 & 23,296 & 38,761 & 30,948 & 23,554 & 38,341 \\
\hline Noviembre 2017 & 25,555 & 18,134 & 32,977 & 25,605 & 18,574 & 32,635 \\
\hline Diciembre 2017 & 18,019 & 12,493 & 23,546 & 18,030 & 12,855 & 23,205 \\
\hline Enero 2018 & 22,182 & 14,814 & 29,551 & 22,474 & 15,336 & 29,613 \\
\hline Febrero 2018 & 19,092 & 12,282 & 25,902 & 19,335 & 12,723 & 25,947 \\
\hline Marzo 2018 & 22,366 & 14,041 & 30,691 & 22,769 & 14,658 & 30,881 \\
\hline Abril 2018 & 27,166 & 16,854 & 37,478 & 27,991 & 17,863 & 38,119 \\
\hline Mayo 2018 & 28,743 & 17,762 & 39,725 & 29,705 & 18,834 & 40,576 \\
\hline Junio 2018 & 25,266 & 15,552 & 34,980 & 26,119 & 16,442 & 35,797 \\
\hline Julio 2018 & 29,165 & 17,650 & 40,680 & 30,250 & 18,781 & 41,720 \\
\hline Agosto 2018 & 33,763 & 20,497 & 47,029 & 35,247 & 21,965 & 48,530 \\
\hline Setiembre 2018 & 30,301 & 18,260 & 42,342 & 31,681 & 19,602 & 43,760 \\
\hline Octubre 2018 & 32,451 & 18,573 & 46,330 & 33,946 & 19,867 & 48,024 \\
\hline Noviembre 2018 & 27,017 & 15,085 & 38,949 & 28,312 & 16,148 & 40,477 \\
\hline Diciembre 2018 & 19,434 & 10,761 & 28,107 & 20,306 & 11,466 & 29,146 \\
\hline Enero 2019 & 23,639 & 12,807 & 34,472 & 24,928 & 13,677 & 36,179 \\
\hline Febrero 2019 & 20,893 & 11,086 & 30,700 & 22,054 & 11,717 & 32,390 \\
\hline Marzo 2019 & 25,427 & 13,291 & 37,563 & 27,024 & 14,097 & 39,950 \\
\hline Abril 2019 & 31,291 & 16,091 & 46,491 & 33,590 & 17,226 & 49,955 \\
\hline Mayo 2019 & 33,298 & 16,869 & 49,727 & 35,869 & 18,115 & 53,623 \\
\hline Junio 2019 & 29,591 & 14,794 & 44,389 & 31,856 & 15,865 & 47,848 \\
\hline Julio 2019 & 35,212 & 17,180 & 53,244 & 38,104 & 18,412 & 57,797 \\
\hline Agosto 2019 & 40,583 & 19,635 & 61,530 & 44,167 & 21,315 & 67,020 \\
\hline Setiembre 2019 & 36,424 & 17,325 & 55,523 & 39,630 & 18,821 & 60,440 \\
\hline
\end{tabular}

Notas: ( ${ }^{*}$ ) Bandas construidas con \pm 2 S.E. al $5 \%$ de significancia

Fuente: Elaborado por los autores

\section{CONCLUSIONES}

El presente trabajo utiliza modelamiento ARIMA de Box \& Jenkins (1976) para el modelamiento y proyección de la demanda de turismo internacional en la región de Puno utilizando información mensual de los años 2003 a 2017 . Utilizando el Criterio de Información de Akaike (AIC) y el Criterio de Schwarz (SC) se seleccionó el modelo SARIMA $(6,1,24)(1,0,1) 12$ como el modelo más eficiente para la demanda del turismo internacional en Puno. Por otro lado, se construyó la Media Porcentual del Error Absoluto (MAPE), el porcentaje de medida del resultado $(Z)$ y el coeficiente de correlación normalizado ( $r$ ) para demostrar la eficiencia de los modelos. El modelo ganador de los cuatro modelos planteados utilizando estos estadísticos es el modelo SARIMA $(6,1,24)(1,0,1) 12$ con pronóstico "bueno" debido que presenta el menor valor del estadístico MAPE igual a 16.15\%. Asimismo, el modelo presenta el mayor valor del porcentaje de medida de resultado $(Z)$ igual a 16.45 y el mayor valor del coeficiente de correlación normalizado r=0.9836. Luego este modelo ganador se puede utilizar para la representación de la demanda de turismo internacional en la región de Puno y su proyección.

Finalmente, los resultados de la presente investigación puede ayudar al sector turismo en la región de Puno y en el Perú para una adecuada planificación y administración de este sector muy importante en la economía.

\section{AGRADECIMIENTOS}

Los autores agradecen a Dios por la guía y bendición. Asimismo, agradecen a los revisores anónimos que contribuyeron con sus aportaciones para la mejora del trabajo. 


\section{REFERENCIAS}

Akaike, H. (1974). A New Look at the Statistical Model Identification. IEEE Transactions on Automatic Control, 19(6), 716-723. https://doi.org/10.1109/TAC.1974.1100705

Akal, M. (2004). Forecasting Turkey's tourism revenues by ARMAX model. Tourism Management, 25(5), 565580. https://doi.org/10.1016/j.tourman.2003.08.001

Athanasopoulos, G., \& Hyndman, R. J. (2008). Modelling and forecasting Australian domestic tourism. Tourism Management, 29 (October 2006), 19-31. https://doi.org/https://doi.org/10.1016/j.tourman.2007.04.009

Baillie, R. (1996). Long Memory Processes and Fractional Integration in Econometrics. Journal of Econometrics, 73(1), 5-59. https://doi.org/10.1016/0304-4076(95)01732-1

Banco Central de Reserva del Perú - BCRP. Gerencia Central de Estudios Económicos (2018). Producto Bruto Interno por sectores productivos (millones S/ 2007). Retrieved March 26, 2019, from https://estadisticas.bcrp.gob.pe/estadisticas/series/anuales/resultados/PM05000AA/html

Banco Central de Reserva del Perú - BCRP. Sucursal Puno. (2018). Síntesis de Actividad Económica, varios meses. Retrieved January 20, 2018, from http://www.bcrp.gob.pe/51-sucursales/sede-regional-puno.html

Botti, L.; Peypoch, N.; Randriamboarison, R.; \& Solonandrasana, B. (2007). An Econometric Model of Tourism Demand in France. Tourismos: An International Multidisciplinary Journal of Tourism, 2(1), 115-126. Retrieved from http://mpra.ub.uni-muenchen.de/25390/

Box, G., \& Jenkins, G. M. (1976). Time Series Analysis: forecasting and control. Oakland, California.

CAMARA. (2018). Cámara de Comercio de Lima - Sector turismo representa 3.3\% del PBI y genera 1.1 millones de empleos. Retrieved from https://www.camaralima.org.pe/repositorioaps/0/0/par/r820 2/informe economico.pdf

Cayo, N., \& Apaza, A. (2017). Evaluación de la ciudad de Puno como destino turístico - Perú. Comuni@cción, 8(2), 116-124. Retrieved from http://www.scielo.org.pe/scielo.php?script=sci arttext\&pid=S221971682017000200005

Chaitip, P., \& Chaiboonsri, C. (2015). Forecasting with X-12-ARIMA y ARFIMA: International Tourist Arrivals to India. Annals of the University of Petroşani, Economics, 9(3), 147-162. Retrieved from http://repositorio.ana.gob.pe/handle/ANA/1564

Chan, F., Lim, C., \& McAleer, M. (2005). Modelling multivariate international tourism demand and volatility. Tourism Management, 26(3), 459-471. https://doi.org/10.1016/j.tourman.2004.02.013

Chang, C., Sriboonchitta, S., \& Wiboonpongse, A. (2009). Modelling and forecasting tourism from East Asia to Thailand under temporal and spatial aggregation, Mathematics and Computers in Simulation, 79, 17301744. https://doi.org/10.1016/j.matcom.2008.09.006

Chokethaworn, K., Wiboonponse, A., Sriboonchitta, S., Sriboonjit, J., Chaiboonsri, C., \& Chaitip, P. (2010). International Tourists' Expenditures in Thailand: A Modelling of the ARFIMA-FIGARCH Approach. The Thailand Econometrics Society, 10(January), 85-98.

Chow, G. (1960). Tests of Equality Between Sets of Coefficients in Two Linear Regressions. Econometrica, 28(3), 591-605. https://doi.org/10.2307/1913018

Chu, F. L. (2008). A Fractionally Integrated Autoregressive Moving Average Approach to Forecasting Tourism Demand. Tourism Management, 29(1), 79-88. https://doi.org/10.1016/j.tourman.2007.04.003

CORREO. (2017). Turismo y empleo en la región Puno. Retrieved March 26, 2019, from https://diariocorreo.pe/peru/turismo-en-la-region-puno-genera-90-mil-empl-7289/

Coshall, J. T. (2009). Combining volatility and smoothing forecasts of UK demand for international tourism. Tourism Management, 30(4), 495-511. https://doi.org/10.1016/j.tourman.2008.10.010

Dickey, D. A., \& Fuller, W. A. (1979). Distribution of the Estimators for Autoregressive Time Series With a Unit Root. Journal of the American Statistical Association, 74(366), 427-431. https://doi.org/10.2307/2286348 
du Preez, J., \& Witt, S. F. (2003). Univariate versus multivariate time series forecasting: An application to international tourism demand. International Journal of Forecasting, 19(3), 435-451. https://doi.org/10.1016/S0169-2070(02)00057-2

Durbin, J., \& Watson, G. S. (1950). Testing for Serial Correlation in Least Squares Regression. I. Biometrika Trust, 58(1), 409-428. https://doi.org/10.2307/2332391

Durbin, J., \& Watson, G. S. (1971). Testing for serial correlation in least squares regression. III. Biometrika, 58(1), 1-19. https://doi.org/10.1093/biomet/58.1.1

GESTION. (2017). Perú: Llegada de turistas por país. Retrieved March 25, 2019, from https://gestion.pe/economia/Ilegaran-4-8-millones-turistas-internacionales-peru-ano-10-2018-258028

Granger, C., \& Joyeux, R. (1980). An Introduction to Long-Memory Time Series Models and Fractional Differencing. Journal of Time Series Analysis, I(1), 15-29. https://doi.org/https://doi.org/10.1111/j.14679892.1980.tb00297.x

Greenidge, K. (2001). Forecasting Tourism Demand An STM Approach. Annals of Tourism Research, 28(1), 98-112. https://doi.org/https://10.1016/S0160-7383(00)00010-4

Hosking, J. R. M. (1981). Fractional differencing. Biometrika, 68(1), 165-176. https://doi.org/10.1093/bio$\underline{\text { met/68.1.165 }}$

Instituto Nacional de Estadística e Informática - INEI (2018a). Pobreza por departamentos del Perú. Retrieved February 4, 2019, from https://www.inei.gob.pe/estadisticas/indice-tematico/living-conditions-and-poverty/ Instituto Nacional de Estadística e Informática - INEI. (2018b). Peru, PBI por departamentos según actividades económicas. Retrieved March 26, 2019, from https://www.inei.gob.pe/estadisticas/indice-tematico/pbi-de-los-departamentos-segun-actividades-economicas-9110/

INRENA. (1995) Evaluacion de la contaminacion del lago titicaca. Retrieved from http://repositorio.ana.gob.pe/handle/ANA/1564

Jarque, C., \& Bera, A. (1980). Efficient tests for normality, homoscedasticity and serial independence of regression residuals. Economics Letters, 6(4), 255-259. https://doi.org/10.1016/0165-1765(81)90035-5

Jarque, C., \& Bera, A. (1981). Efficient tests for normality, homoscedasticity and serial independence of regression residuals Monte Carlo Evidence. Journal of the American Statistical Association, 7, 313-318. https://doi.org/doi:10.1016/0165-1765(81)90035-5

Jarque, C., \& Bera, A. (1987). A test for Normality of observations and Regression Residuals. International Statistical Review, 55(2), 163-172. https://doi.org/10.2307/1403192

Kwiatkowski, D.; Phillips, P.; Schmidt, P.; \& Shin, Y. (1992). Testing the null hypothesis of stationarity against the alternative of a unit root. Journal of Econometrics, 54(1-3), 159-178. https://doi.org/10.1016/03044076(92)90104-Y

Laurente, L. \& Poma, R. (2016). Introducción a la teoría de las probabilidades (Primera Ed). Puno, Perú.

Law, R., \& Au, N. (1999). A neural network model to forecast Japanese demand for travel to Hong Kong. Tourism Management, 20(1), 89-97. https://doi.org/10.1016/S0261-5177(98)00094-6

Lee, C. K.; Song, H. J.; \& Mjelde, J. W. (2008). The forecasting of International Expo tourism using quantitative and qualitative techniques. Tourism Management, 29(6), 1084-1098. https://doi.org/10.1016/j.tourman.2008.02.007

Lewis, C. D. (1982). Industrial and business forecasting methods. London: Butterworths. Retrieved from http://interstat.statjournals.net/YEAR/2008/articles/0810005.pdf

Lim, C., \& Mcaleer, M. (1999). A seasonal analysis of Malaysian tourist arrivals to Australia. Mathematics and Computers in Simulation, 48(4), 573-583. https://doi.org/https://10.1016/S0378-4754(99)00038-5

Ljung, G., \& Box, G. (1978). Biometrika Trust On a Measure of Lack of Fit in Time Series Models. Biometrika, 65(2), 297-303. https://doi.org/https://doi.org/10.1093/biomet/65.2.297

Loganathan, N., \& Ibrahim, Y. (2010). Forecasting International Tourism Demand in Malaysia Using Box Jenkins Sarima Application. South Asian Journal of Tourism and Heritage, 3(2), 50-60.

Mackinnon, J. (1996). Numerical Distribution Functions for Unit Root and Cointegration Tests. Journal of Applied Econometrics, 11(6), 601-618. $\quad$ https://doi.org/https://doi.org/10.1002/(SICl)10991255(199611)11:6<601::AID-JAE417>3.0.C0;2-T 
Mamani, L. (2016). Impacto socioeconómico del turismo rural comunitario de Karina-Chucuito. Universidad Nacional del Altiplano. Retrieved from http://repositorio.unap.edu.pe/bitstream/handle/UNAP/1802/Articulo.pdf?sequence=1\&isAllowed=y

MINCETUR. (2017a). Perú: Arribo de visitantes extranjeros a establecimientos de hospedaje, según región. Serie estadística 2003-2017. Retrieved January 17, 2018, from http://datosturismo.mincetur.gob.pe/appdatosTurismo/Content3.html

MINCETUR. (2017b). Perú: Llegada de turistas internacionales según país de residencia permanente. Retrieved March 25, 2019, from http://datosturismo.mincetur.gob.pe/appdatosTurismo/Content1.html

MiViaje. (2018). Descubre las islas de los uros en el lago Titicacaca - Panel fotográfico. Retrieved March 25, 2019, from https://miviaje.com/las-islas-de-los-uros-lago-titicaca/

Nanthakumar, L., Subramaniam, T., \& Kogid, M. (2012). Is “Malaysia Truly Asia”? Forecasting tourism demand from ASEAN using SARIMA approach. Tourismos, 7(1), 367-381. Retrieved from http://www.chios.aegean.gr/tourism/VOLUME 7 No1 art20.pdf

Organización Mundial del Turismo - OMT. (2017). Panorama OMT del turismo internacional. Retrieved from http://www.e-unwto.org/doi/book/10.18111/9789284419043

Peiris, H. (2016). A Seasonal ARIMA Model of Tourism Forecasting: The Case of Sri Lanka. Journal of Tourism, Hospitality and Sports, 22, 98-109. Retrieved from https://www.iiste.org/Journals/index.php/JTHS/article/view/33831

Peiris, M. S., \& Perera, B. J. C. (1988). On Prediction with Fractionally Differenced Arima Models. Journal of Time Series Analysis, 9(3), 215-220. https://doi.org/10.1111/j.1467-9892.1988.tb00465.x

Phillips, P. C., \& Perron, P. (1988). Testing for a unit root in time series regression. Biometrika, 75(2), 335346. https://doi.org/10.1093/biomet/75.2.335

Psillakis, Z., Alkiviadis, P., \& Kanellopoulos, D. (2009). Low cost inferential forecasting and tourism demand in accommodation industry. Tourismos: An International Multidisciplinary Journal of Tourism, 4(2), 47-68.

PUNO. (2017). Principales recursos turísticos em Puno. Retrieved from http://www.bcrp.gob.pe/docs/Sucursales/Puno/Puno-Atractivos.pdf

Ray, B. K. (1993). Modeling Long Memory Processes for Optimal Long Range Prediction. Journal of Time Series Analysis, 14(5), 511-525. https://doi.org/10.1111/j.1467-9892.1993.tb00161.x

Reisen, V. (1994). Estimation of the fractional difference parameter in the ARIMA (p,d,q) model using the smoothed periodogram. Journal of Time Series Analysis, 15(3), 335-350. https://doi.org/https://doi.org/10.1111/j.1467-9892.1994.tb00198.x

Schwarz, G. (1978). Estimating the Dimension of a Model. The Annals of Statistics, 6(2), 461-464. https://doi.org/10.1214/aos/1176344136

Shareef, R., \& McAleer, M. (2005). Modelling International Tourism Demand and Volatility in Small Island Tourism Economies. International Journal of Tourism Research, 7(6), 313-333. https://doi.org/10.1002/jtr.538

Shitan, M. (2008). Time series modelling of tourist arrivals to Malaysia. Department of Mathematics, Faculty of Science, and Applied \& Computational Statistics Laboratory, Institute for Mathematical Research, Universiti Putra Malaysia. Retrieved from http://interstat.statjournals.net/YEAR/2008/articles/0810005.pdf

Zhou, T., Bonham, C., \& Gangnes, B. (2007). Modeling the supply and demand for tourism: a fully identified VECM approach. Department of Economics and University of Hawaii Economic Reserch Organization. University of Hawaii at Manoa. Working Paper No. 07-17, July 20, 2007. https://doi.org/10.1001/archinte.165.8.854 


\section{Informaciones de los autores}

\section{Luis Francisco Laurente Blanco}

Ingeniero Economista por la Universidad Nacional del Altiplano con Maestría en Informática por la misma universidad, con estudio de posgrado en matemáticas y estadística en la USP e IMPA del Brasil, su área de interés es la economía matemática. Actualmente realiza investigaciones en el Grupo Fibonacci de Ciencias Económicas (GRFICE) con varios libros y artículos publicados.

Contribuyó en el artículo en: revisión de la literatura, búsqueda de información, análisis de datos y discusión.

E-mail: flaurenteblanco@gmail.com

ORCID: https://orcid.org/0000-0002-8531-3959

\section{Ronald Wilson Machaca Hancco}

Ingeniero Economista por la Universidad Nacional del Altiplano es Coordinador de Nivelación Escolar Voluntades en el Centro de Atención Residencial Sagrado Corazón de Jesús.

Contribuyó en el artículo en: revisión de la literatura, conclusiones y discusión.

E-mail: karegarou@gmail.com@gmail.com

ORCID: https://orcid.org/0000-0002-4035-5581 